\title{
Logística Urbana desde la perspectiva del Agente Generador de Viajes
}

\section{Urban Logistics from the perspective of the Travel Generator Agent}

\author{
DOI: http://dx.doi.org/10.17981/ingecuc.15.1.2019.05
}

Artículo de Investigación Científica. Fecha de Recepción: 31/07/2018 Fecha de Aceptación: 12/01/2019

\section{Oscar Palacio-León}

Universidad Militar Nueva Granada. Bogotá D.C, (Colombia)

oscar.palacio@unimilitar.edu.co

Para citar este artículo:

Oscar Palacio-León, "Logística Urbana desde la perspectiva del Agente Generador de Viajes", INGE CUC, vol. 15, no. 1, pp. 45-62, 2019. DOI: http://doi.org/10.17981/ingecuc.15.1.2019.05

\section{Resumen}

Introducción- La logística urbana es la encargada de planificar y gestionar los servicios de una ciudad, a fin de atraer inversiones y retener actividades productivas. En donde, las estrategias de negocio del tipo ganar-ganar, son uno de los tantos medios útiles con que cuenta la administración de la ciudad, para viabilizar la retención de empresas extendidas productivas y de servicio, que se pueden estructurar desde la gestión del inventario conjunto, siguiendo uno de tres enfoque de dirección, a saber: i) Agente generador de carga; ii) Agente regulador de carga; y iii) Agente generador de viajes. El presente trabajo, aborda el último enfoque de dirección ambientado en una economía compartida en estado de incertidumbre y ambigüedad, por ser un esquema empresarial urbano, poco documentado en la literatura científica fáctica.

Objetivo- Diseñar una estrategia de administración del inventario conjunto en estado de incertidumbre y ambigüedad, con respecto al agente generador de viajes en la empresa extendida urbana de economía compartida.

Metodología- El desarrollo de la investigación se llevó a cabo mediante la ejecución de una estructura operacional de analisis de eficiencia técnico-económica y financiera, con la que se llevó a cabo el diseño de la estrategia de administración del inventario conjunto en condiciones de incertidumbre y ambigüedad, a fin de identificar los aspectos en que influye el agente generador de viajes en la empresa extendida urbana de economía compartida.

Resultados-Con el análisis de la eficiencia técnico-económica y financiera obtenida se demuestra que, una forma de gestionar el inventario conjunto en estado de incertidumbre y ambigüedad, es el cálculo centrado en la econometría con aplicación en los juegos de transferencia de utilidad, basando su viabilidad, elegibilidad y prioridad en las matemáticas financieras borrosas.

Conclusiones- Por lo general, la gestión del inventario conjunto se da en el marco de la negociación concretada, entre los Agentes Generadores tanto de Carga como de Viajes, a fin de balancear en equidad las Estrategias contrapuestas Push y Pull, en que soportan su integración como empresa extendida urbana.

Palabras clave- Empresa Extendida; Agente Generador de Viajes; Unidad de Carga Eficiente; Óptimo Social; Creación de Valor Compartido; Matemáticas financieras borrosas.

\begin{abstract}
Introduction- Urban logistics has been responsible for planning and managing the services of a city with the purpose of attracting investments and retaining productive activities. Where, the strategies of business of the type to win to win, are one of so many useful means of the administration of the city, on the viability of the retention of widespread, productive companies and of service, which can be structured from the management of the joint inventory, following one of three approaches of direction: i) Load generator agent, ii) Load regulating agent, and iii) Generating agent of trips. Since the third perspective has been documented the present work, about a shared economy in a state of uncertainty and ambiguity, because it is an urban business scheme, little documented in scientific literature.
\end{abstract}

Objective- To design a strategy of administration of the joint inventory in a condition of uncertainty and ambiguity, with regard to the generating agent of trips in the widespread urban company of shared economy.

Methodology - The development of the research was carried out through of the execution of an operational structure of analysis of technical - economic and financial efficiency, with which there was carried out the design of the strategy of administration of the joint inventory in conditions of uncertainty and ambiguity, in order to identify the aspects in which the generating agent of trips influences the widespread urban company of shared economy.

Results- With the analysis of the technical-economic and financial efficiency obtained, it is demonstrated that one way to manage the joint inventory in a state of uncertainty and ambiguity is the calculation centered on econometrics with application in utility transfer sets, basing its viability, eligibility, and priority on Fuzzy financial math.

Conclusions- In general, the management of the joint inventory takes place within the framework of the concrete negotiation, between the load generator agent as in the of trips in order to balance in equity opposed Push and Pull Strategies, in which they support their integration as an urban extended company.

Keywords- Extended Enterprise; Travel Generator Agent; Efficient Load Unit; Optimal Social; Creation of Shared Value; Fuzzy financial math. 


\section{INTRODUCCIÓN}

Adarme-Jaimes [1], sostiene que "la logística surge de la necesidad no solo de movilizar personas sino también de mover y/o almacenar productos e información a nivel tanto intraempresa como interempresa". Más aun, Chopra y Meindl [8], consideran los siguientes drivers para su desarrollo: i) Infraestructura; ii) Tecnologías de la Información y Comunicaciones; iii) Institucionalidad; iv) Procesos; v) Personas; y vi) Fijación de Precios. En tal sentido, el laboratorio natural de la logística es la cadena de suministros multinivel (Fig. 1).

Tal como se ilustra en la Fig. 1, el escenario en el cual se generan, no solo de forma incierta, sino también compleja las relaciones entre los agentes descritos es la ciudad [1]-[9]-[21]. En la actualidad las ciudades de América Latina y el Caribe albergan en sus principales ciudades el $80 \%$ de su población [22], al punto de que algunas de ellas se consideran megaciudades, como es el caso de Río de Janeiro, Buenos Aires o Sao Paulo, ciudades con más de 10 millones de habitantes [22]-[28]. Hay que mencionar, además que la ciudad es la unidad básica de la economía de una nación, que no sólo debe ser sostenible desde lo ambiental sino también inclusiva desde el punto de vista de lo social [1]-[30].

Así mismo, el documento CONPES $3819^{1}$, define Sistema de Ciudades como "un conjunto organizado de ciudades que comparten relaciones funcionales de orden económico, social, cultural y ambiental, y que interactúan entre sí, para maximizar los beneficios de la urbanización y minimizar los costos sociales" [11]. Documento que establece la Política Nacional que promueve, por un lado, y fortalece, por el otro, el estudio de la Logística Urbana por parte de la Universidad, el Estado y la Empresa en todo el territorio de Colombia, siendo un buen ejemplo a referir sobre su puesta en marcha al día de hoy, el proyecto financiado con regalías asignadas por la nación a la Alcaldía Mayor de Bogotá D.C y a la Gobernación de Cundinamarca, que lleva por título: Corredor Tecnológico Agroindustrial [1].

Por otro lado, Taniguchi, Fwa y Thompson definen la Logística Urbana como "un proceso de total optimización de las actividades logísticas y de transporte llevadas a cabo por la empresa privada en zonas urbanas, teniendo en cuenta factores como el tráfico, congestión y consumo de energía, en el marco de una economía de mercado" [34]. Cabe resaltar los aportes de Gonzalez-Feliu, Semet y Routhier, al sostener que...

... la logística urbana es un campo multidisciplinario que tiene como objetivo comprender, estudiar y analizar las diferentes organizaciones, los sistemas logísticos, los interesados y las acciones de planificación relacionadas con la mejora de los diferentes sistemas de transporte de mercancías en una zona urbana y vincularlos de forma sinérgica" [19].

Más no se trata tan sólo de ver la Logística Urbana desde su concepción, sino de su evolución como inductor de Creación de Valor Compartido (CVC), desde

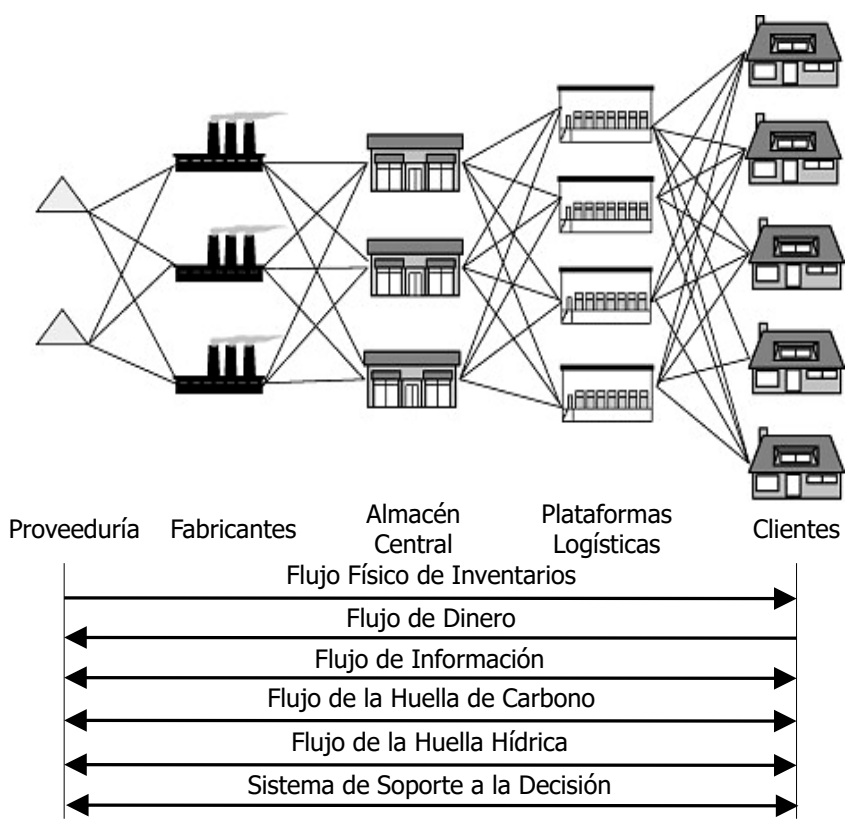

Fig. 1. Cadena de Suministros.

Fuente: Adaptado de [1].

${ }^{1}$ Política nacional para consolidar el sistema de ciudades en

Colombia. Conpes 3819. 21-10-2014 
la óptica del Agente Generador de Viajes (AGV), en atención al nivel de integración tanto vertical como horizontal que sinergia el diseño de una Red de Suministro Urbana, en particular [30]. Por ende, el presente artículo tiene como propósito diseñar una estrategia de gestión del inventario conjunto con respecto al AGV, para una Empresa Extendida de Economía Compartida en condiciones de incertidumbre e ambigüedad.

El presente manuscrito se ha organizado con la siguiente estructura: En la Sección 2, Revisión Literaria, se aborda la integración de la Empresa Extendida de Economía Compartida $\left(\mathrm{EE}_{\mathrm{EC}}\right)$, desde la óptica del AGV, por un lado, con un enfoque en la gestión del inventario conjunto, por el otro. En la Sección 3, Metodología, se describe la Metodología de Integración y Encadenamientos Logísticos para la Creación de Valor Compartido (MIEL ${ }_{\mathrm{CVC}}$ ), en que se soporta el mecanismo de CVC en condiciones de incertidumbre e ambigüedad desde la perspectiva del AGV, con enfoque en la Unidad de Carga Eficiente (UCE). En la Sección 4, Resultados, se encontró que una estrategia de integración del tipo ganar-ganar puede constituirse no solo por una recompensa en equilibrio sostenible sino también por un óptimo social, cuando se desea configura una red de suministros urbana. Finalmente, en la Sección 5, Conclusión, se presentan los principios, relaciones y generalizaciones que arrojan los resultados obtenidos de la investigación, contrastado con el objetivo propuesto.

\section{REVISIÓN LITERARIA}

En esta sección, se presentan los elementos constitutivos de la estrategia de gestión del inventario conjunto con enfoque en el $\mathrm{AGV}$, para una $\mathrm{EE}_{\mathrm{EC}}$ en condiciones tanto de incertidumbre como de ambigüedad.

\section{A. Empresa Extendida}

El concepto de Empresa Extendida (EE) consiste en integrar en la estrategia de negocio a todos los agentes que hacen parte, de principio a fin, de la Red de Suministros [5], dado uno de los enfoques estratégicos de diseño, que a continuación se citan: i) Logística por Conglomerados; ii) Logística de Economía Compartida; iii) Logística Justa; y iv) Logística Omnicanal [30].

La EE es el medio que amplía a una organización no sólo en espacio sino también en tiempo, a fin de incrementar así su eficiencia, al atreverse como sistema productivo a tomar ventaja del riesgo para aprovecharlo como una oportunidad clave para el diseño de aspectos estratégicos, operativos e innovadores, que la hagan competitiva en forma sostenible (Fig. 2), durante la vigencia del Ciclo de Inversión y Financiamiento (CIF) que da soporte al acuerdo no sólo contractual sino también vinculante entre sus agentes [31].

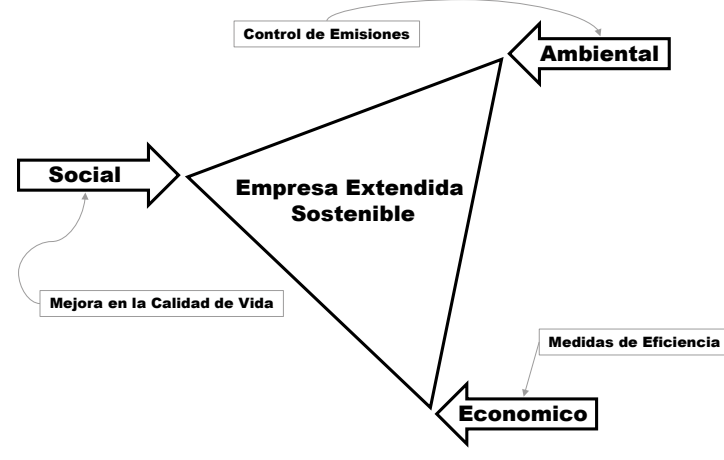

Fig. 2. Arquitectura Empresarial Sostenible. Fuente: Adaptado de [1]

El paradigma holónico es uno de los enfoques en el ámbito de la Ingenieria Organizacional, que permite que la EE como sistema empresarial sea viable, desde el punto de vista de la Arquitectura Empresarial Sostenible (Fig. 2), a la vez, que hace de la integración de sus agentes una relación duradera y estable a nivel estratégico [5]-[32].

Es decir, la EE está conformada por agentes (Holones) interrelacionados entre sí, que conforma una unidad básica de negocio orientada a la maximización del desempeño de las capacidades de sus recursos, a la vez, que CVC para sus interesados [2]-[3], en donde, la Fig. 3 recoge el aporte que hace el paradigma holónico a la Red de Suministros Urbana.

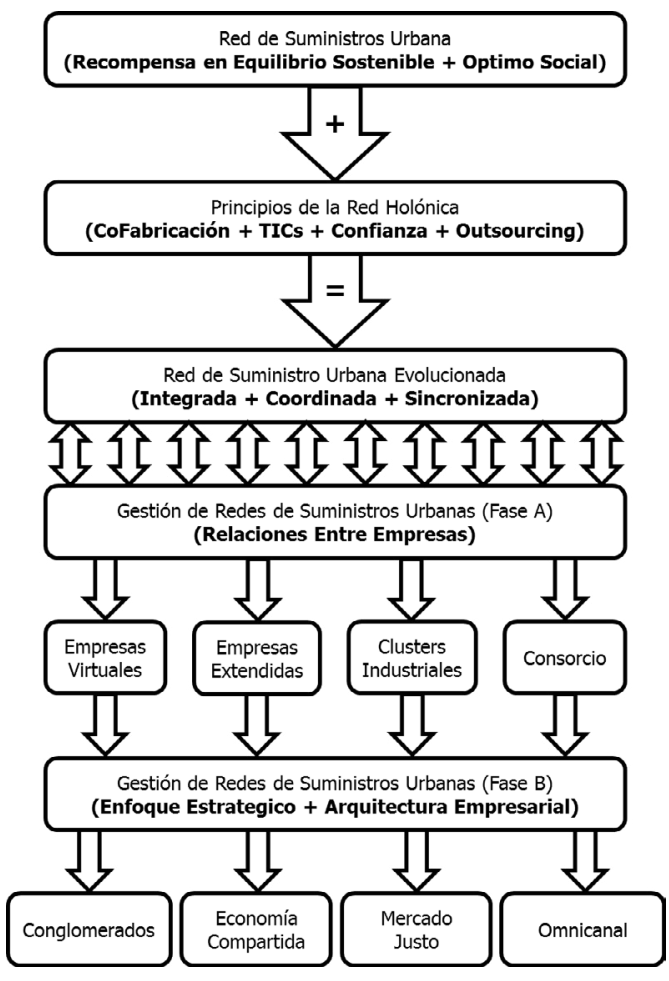

Fig. 3. Logística Urbana Holónica. Fuente: Adaptado de [30]. 
Conviene subrayar que la Red de Suministros Urbana con enfoque holónico, se adapta con facilidad a la dinámica del mercado, permitiendo la continuidad del negocio en forma sostenible, al hacer tanto de la colaboración como de la cooperación de sus agentes, una palanca de flexibilidad operativa en la toma de decisiones vía reglas consensuadas, que restringe su autonomía de actuación, toda vez que, les asegura desempeños tanto controlables como predecibles, en función al uso racional de sus recursos como del tiempo de respuesta [2]-[3]-[30].

Por otra parte, el paradigma holónico permite construir el patrón de conducta de la EE en atención a la ambigüedad de tipo [3]: i) Organizacional; ii) Variedad Requerida; y iii) Sostenibilidad; En consonancia con la evolución del entorno de negocio, por medio de [3]: i) La aplicación de sus principios y propiedades; ii) El ciclo de vida del holón; iii) El diseño de mecanismos de autorregulación del holón; y iv) La Resiliencia holónica. En los siguientes apartados se hace una contextualización de este paradigma, frente al diseño de una Estrategia de Negocio del Tipo Ganar-Ganar, que CVC, por un lado, y brinda un Óptimo Social, por el otro, a los agentes que conforman la $\mathrm{EE}_{\mathrm{EC}}$

\section{B. Agente Generador de Viajes}

La Distribución Urbana Mercancías (DUM) involucra los movimientos relacionados con el suministro de bienes de consumo, paquetería industrial y carga en general, en los contextos que a continuación se citan: i) Sistemas de ciudades; ii) Distribución Capilar; y iii) Centros Históricos [1]-[30]. La DUM es una herramienta de planificación estratégica tanto de la economía como de la política pública de ciudad, que permite definir el Perfil Logístico Urbano en atención, bien sea, del Agente Generador de Carga (AGC), Agente Regulador de Carga (ARC) o con respecto al $A G V$, sobre quienes recae el diseño del Sistema de Soporte a la Decisión (SSD) del Transporte, valuado por lo general, con base en factores dimensionales como peso, volumen, número de viajes, entre otros [1]-[8]-[19]-[30].

En contraste con lo anterior, es apropiado mencionar que el estudio de Demanda de Transporte de Carga en Áreas Urbanas $\left(\mathrm{D}_{\mathrm{T}} \mathrm{Q}_{\mathrm{U}}\right)$ [7], por lo general, están soportados en la modelación tradicional que incluyen: i) Modelos Directos de Demanda; ii) Variantes del Modelo de Cuatro Pasos; y iii) Modelos Insumo-Producto; los cuales, pueden utilizar herramientas como: Encuestas Origen-Destino, Encuesta Regional de Desempeño del Transporte Automotor de Carga (TAC), entre otras [12]-[20]-[29]-[36].

Conviene subrayar que en la década del 70 se hicieron los dos primeros estudios cuantitativos, con relación a la $\mathrm{D}_{\mathrm{T}} \mathrm{Q}_{\mathrm{U}}$ con respecto al AGV. La primera investigación en esta materia fue desarrollada por Smith [7], en la cual, se caracterizó los viajes urbanos por camión en relación con datos provenientes de la compañía o de su ubicación. Mientras que el segundo trabajo, lo abordo Hutchinson [7], quien planteo una serie de ecuaciones de proyección de dicha demanda, a través, de la generación de los viajes requeridos por el sistema productivo con base en el número de camiones.

Otro rasgo de los SSD de la $\mathrm{D}_{\mathrm{T}} \mathrm{Q}_{\mathrm{U}}$ con enfoque en el AGV se originó en la década del 90, con el desarrollo de tres nuevos trabajos. En donde, el primero de ellos categorizo la DUM con respecto a la demanda en [7], [12]: Modelos basados en la generación de la carga (Mercancías) y Modelos basados en la generación de viajes (Camión). El segundo, entretanto fue propuesto por el Departamento de Transportes de los Estados Unidos, que buscaba difundir un protocolo básico para prever cambios en la demanda por carga, con base en alteraciones en el nivel de actividades económicas entre otros factores relacionados [7][29]. En último lugar, se encuentra el trabajo de [7], quienes asocian al modelado de la demanda agregada de carga, la inclusión de la capacidad disponible de bodegaje por zonas urbanas.

Hay que mencionar, además que en la primera década del siglo XXI, se perfecciono la metodología de los cuatro pasos usada en la modelación de la demanda de transporte de pasajeros, para ser aplicado por el SSD de la $\mathrm{D}_{\mathrm{T}} \mathrm{Q}_{\mathrm{U}}$ con enfoque en el AGV, como se indica en la Fig. 4.

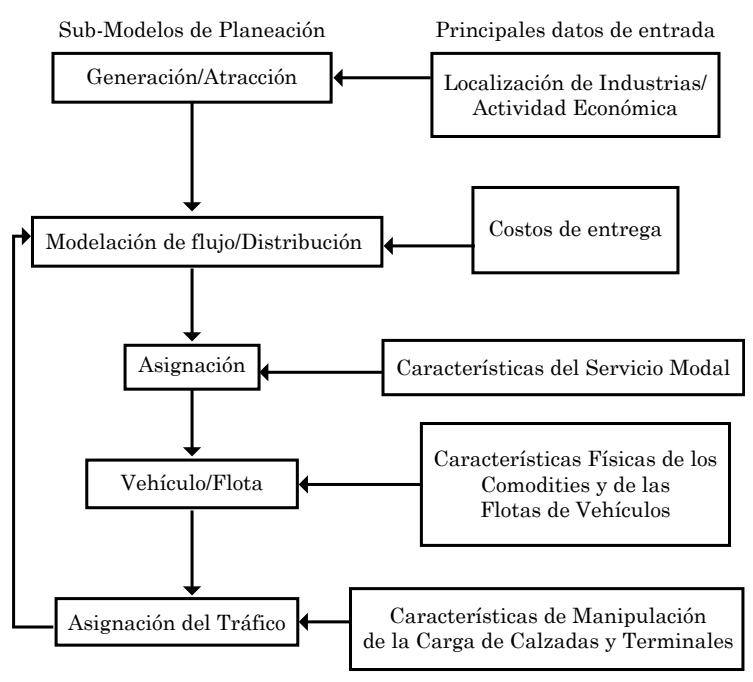

Fig. 4. Metodología Multipasos DTQU. Fuente: Adaptado por [20].

La metodología descrita en la figura 4, está conformada por métodos, herramientas y técnicas aportadas por [7], [12], [20], [29], [36]. Por lo demás, [20] adapto y aplico esta metodología, para modelar a nivel estratégico el transporte de carga a escala interregional en Colombia con enfoque en el análisis de productos. Siendo su aporte la mejora del SSD dadas algunas variantes necesarias, tales como: "la estimación de los vehículos vacíos y la conversión a 
flujo vehicular", que permita adquirir información importante, confiable y fidedigna para los agentes que constituyen la $\mathrm{EE}_{\mathrm{EC}}$.

Así mismo, en la segunda década del siglo XXI se ha estado perfeccionando el SSD de la $\mathrm{D}_{\mathrm{T}} \mathrm{Q}_{\mathrm{U}}$ con enfoque en el $\mathrm{AGV}$, con respecto a la depuración y validación de las variables económicas que explican mejor la generación de viajes en zonas urbanas, por concepto de demanda de carga consolidada, cuyos resultados indican que el área del establecimiento comercial contribuye también con dicha explicación [12]-[29]-[36]. A su vez, se ha modelado esquemas de DUM tipo Hub-and-Spoke (DUM HAS $_{\text {}}$ ) para economía compartida bajo dos estrategias, a saber [24]: i) Operación de Parada de Taxi (OPT); y ii) Operaciones de Paradas de Autobús (OPA).

Cabe resaltar que el DUMHAS busca mitigar el tránsito de vehículos de carga vacíos, mejorando a su vez los tiempos de respuesta de los eslabones proveedor-comprador, entre otras medidas de rendimiento de la EE [38]. Sin embargo, esta investigación a la fecha no ha podido evidenciar la existencia de una metodología con fundamento en el paradigma holónico, que configure una EEEC bajo una estrategia de integración del tipo Ganar-Ganar desde la perspectiva del AGV, basada en el área del establecimiento comercial bajo un planeamiento de DUMHAS, por medio de la cual, sea gestionada la UCE en condiciones de Incertidumbre e Ambigüedad.

\section{Gestión del Inventario Conjunto}

El paradigma holónico contribuye a la configuración de $\mathrm{EE}_{\mathrm{EC}}$ (figura 3), la cual, posee la capacidad de proveer en las condiciones adecuadas, el producto correcto, en la cantidad requerida, en el lugar preciso, en el tiempo pactado, a un costo razonable y a entera satisfacción del receptor [30]. Más aún, se constituye en una estructura operativa que aporta los instrumentos necesarios, para construir y sostener ventajas gana-gana efectivas por parte de la coalición de agentes, que a través, de un Acuerdo Contractual y Vinculante buscan integrar, sincronizar y coordinar los flujos de información, materiales, huella hídrica, huella de carbón, de reversa y dinero tanto a nivel intra como inter empresa, bajo un SSD basado en la Gestión del Inventario Conjunto [30].

La Gestión del Inventario Conjunto, se característica por fomentar las buenas prácticas empresariales en mejora de la red de suministros urbana, dada el diseño de una política común, sostenible y equilibrada a nivel estratégico entre los agentes que la conformen [30]. Ya que, se dan en la EE que presentan estrategias y acciones dirigidas a lograr la CVC, siendo necesario el delinear un mecanismo por medio del cual, se articule la política con las estrategias que propendan por la rentabilización bajo un óptimo social de la EE, en donde la UCE coadyuva con este propósito [30].
Se debe agregar que el diseño de la Red de Suministros en su eje Horizontal, ha sido soportado en Analisis de Eficiencia Técnico-Económica, no sólo por académicos sino también por empresarios, vinculada a Estrategias de Coordinación del Inventario Conjunto, tales como [1]-[30]: i) Desarrollo Conjunto de Órdenes (DCO); ii) Justo a Tiempo (JIT: Just in Time); iii) Respuesta Rápida (QR: Quick Response); iv) Reaprovisionamiento Continuo (CR: Continuous Replenishment); v) Inventario Administrado por el Proveedor (VMI: Vendor Managed Inventory); vi) Gestión de la Disponibilidad por el Proveedor (SMA: Supplier Managed Availability); vii) Épocas Comunes de Resurtido (CRE: Common Replenishment Epochs); viii) Planeación, Pronóstico y Reabastecimiento Colaborativo (CPFR: Collaborative Planning, Forecasting and Replenishment); y ix) Reaprovisionamiento Eficiente (ER: Efficient Replenishment).

Sin embargo, esta investigación no pudo evidenciar que las estrategias citadas en el párrafo anterior, hubiesen sido usadas para configurar una Empresa Extendida Urbana de Economía Compartida con enfoque en el AGV, por un lado, que gestionen el inventario conjunto en condiciones de incertidumbre y ambigüedad, por el otro. Razón por la cual, se diseñó una estrategia de integración del tipo ganarganar para la EE, descrita en la Fig. 5, la cual, se basa en la UCE en Condiciones de Incertidumbre y Ambigüedad.

En donde, la UCE es un mecanismo de CVC soportado en la mejor estrategia de integración del tipo ganar-ganar, por medio del cual, se potencializa capacidades en la EE dada la unitarizacion de la carga, en especial, cuando se relaciona con productos no perecederos [30].

En otras palabras, la UCE permite la integración de la EE en lo referente a los ámbitos de orden funcional, espacial, intertemporal y empresarial; con miras a mejorar desde los lineamientos del paradigma holónico su desempeño en lo económico, social y ambiental [2]-[3]-[30]-[32]. Además, garantiza a cada uno de los agentes que la conforman su autonomía administrativa, independencia económica y respecto por su cultural e idiosincrasia organizacional [30], a la vez, que facilita de forma eficiente las operaciones de manipulación, bodegaje y transporte del inventario de consumo [26]-[30].

Se debe agregar que la UCE aporta al estudio de la $D_{T} Q_{U}$ con relación a: i) El Diseño de Tamaños Modulares; ii) El incremento de productividad e reducción de costos; y iii) El funcionamiento de los embalajes en términos de la resistencia que ofrece a sus contenidos cuando son sometidos a vibraciones de baja frecuencia [26]-[30]. No obstante, la dificultad que afronta la UCE estriba en su estandarización en cuanto a formas y cubicaje, por ser un mecanismo de CVC [30], que todavía está en permanente construcción [26]. 
Desde otro punto de vista, la UCE permite el estudio de la integración de la $\mathrm{EE}_{\mathrm{EC}}$ a nivel no sólo vertical sino también horizontal [30], bajo un planeamiento de la $\mathrm{D}_{\mathrm{T}} \mathrm{Q}_{\mathrm{U}}$ limitada al área del establecimiento comercial, en atención a los lineamientos de la DUM $_{\text {HAS }}$ basados en el paradigma holónico en condiciones de incertidumbre e ambigüedad con enfoque en el $\mathrm{AGV}$, cuya metodología se expone en el presente trabajo.

\section{Logística de economía compartida}

Tal como se mencionó anteriormente, la unidad básica de análisis de la Red de Suministros Urbana es la EE. En tal sentido, para el caso específico de la $\mathrm{EE}_{\mathrm{EC}}$, la UCE se constituye en su unidad básica de análisis de integración tanto vertical como horizontal, dada la gestión conjunta del inventario de consumo [30]. El anterior concepto se esclarecerá en lo que sigue, la UCE es el resultado de negociar el mejor diseño de carga unitarizada entre los agentes de la EE que supere, por un lado, sus particularidades, y, por el otro, rentabilice las actividades de transporte, bodegaje y manipulación, en atención a su uso equilibrado y racional [26]-[30].

Con esto se quiere decir, que la integración de una EEEC, es el resultado de incluir en su estrategia de negocios criterios de sostenibilidad (Fig. 2), con la meta de asegurar en el largo plazo beneficios para sus interesados [31], que para la presente investigación fueron valorados en un horizonte de cinco años, con una tasa de oportunidad que involucra la tasa social de descuento de Colombia, que el paradigma holónico la configura como una organización inteligente frente al riesgo, la cual, diseña la UCE en condiciones de incertidumbre y ambigüedad con enfoque en el AGV, bajo un planeamiento de la $\mathrm{D}_{\mathrm{T}} \mathrm{Q}_{\mathrm{U}}$ limitada al área del establecimiento comercial, en atención a los lineamientos de la $\mathrm{DUM}_{\mathrm{HAS}}$, como se indica en las próximas secciones.

\section{Metodología}

MIEL $_{\mathrm{CVC}}$ está concebida en los principios del paradigma holónico para la $\mathrm{EE}$, que permite diseñar estrategias de integración del tipo ganar-ganar en función de la UCE en Condiciones de Incertidumbre y Ambigüedad, que consta de las tres etapas que se citan y describen a continuación: A) Herramienta de captura de información en la EE; B) Formulación de la UCE en la EE; y C) Evaluación técnico-económica de UCE en la EE.

\section{A. Herramienta de captura de información en EE}

El SSD de MIEL ${ }_{\text {CVC }}$ está estructurado en el Método SCAMPER para la resolución de problemas en forma creativa, basándose en una lista de chequeo frente a las siguientes acciones: i) Sustituir; ii) Combinar; iii)
Adaptar; iv) Modificar; v) Permutar; vi) Eliminar; y vii) Reordenar [33]. Método que vincula herramientas computacionales de búsqueda, captura y procesamiento de datos e información, por medio de las cuales, se corren las técnicas matemáticas que soportan los modelos cuantitativos que se exponen en los ítems subsiguientes de esta metodología, a fin de diseñar la mejor estrategia de tipo ganar-ganar, en la que se configura la $\mathrm{EE}_{\mathrm{EC}}$ de enfoque y características citadas en párrafos anteriores.

\section{B. Formulación de la $U C E_{M}$ en $E E$}

La UCE es un mecanismo de CVC que permite integrar la EE en sus dos ejes de gestión, a saber: i) Horizontal; y ii) Vertical. La UCE en el plano horizontal se desarrolla con estrategias de colaboración, mientras que en el vertical se hace mediante estrategias de cooperación, para los dos casos se emplean las del tipo ganar-ganar [30]. Siendo una particularidad de la integración horizontal, los esquemas de gestión del inventario entre sus agentes, mientras el AGC emplea el enfoque Push aguas debajo de EE, AGV utiliza el Pull aguas arriba de la misma [1]-[30].

Se debe agregar que mientras que el AGC desea modelos de inventario que maximicen el uso de su capacidad de producción del tipo justo a tiempo, el AGV desea los del tipo cantidad económica de pedido [1]-[30]. Dicho de otra manera, sus objetivos son contrapuestos con respecto a la gestión del inventario conjunto, generando la necesidad de llegar a unos acuerdos contractuales y vinculantes entre los agentes de la $\mathrm{EE}_{\mathrm{EC}}$, a través del diseño de una política de largo plazo que sea común, sostenible y equilibrada al respecto [30].

En coherencia con las especificaciones metodológicas formuladas, a continuación se presenta un primer esbozo correspondiente al diseño de un mecanismo de CVC, que involucra esta caracterización, desde el planeamiento de la $\mathrm{D}_{\mathrm{T}} \mathrm{Q}_{\mathrm{U}}$ con enfoque en el $\mathrm{AGV}$, bajo los lineamientos de la DUM $\mathrm{DAS}_{\mathrm{HAS}}$ con limitación al área del establecimiento comercial, que brinde a la $\mathrm{UCE}_{\mathrm{M}}$ una textura cuantitativa de diseño a partir de una estrategia del tipo ganar-ganar, por un lado, a la vez, que facilita el desarrollo de ordenes conjuntas de pedido en la $\mathrm{EE}_{\mathrm{EC}}$ en condiciones de incertidumbre y ambigüedad, por el otro, [30]-[31]-[38].

La Fig. 5, representa una EEEC estructurada por Un AGC con Un ARC con Múltiples AGV para Gestionar Múltiples Productos. La $\mathrm{UCE}_{\mathrm{M}}$ como mecanismo de CVC se construye en dos etapas. La primera, se ejecuta entre el AGC y el ARC, denominada Inbound. La segunda, implica coordinar la Estrategia Ganar-Ganar del AGV aguas arriba de la Outbound, en función a la mejora de la EE vía Óptimo Social (Modelo Cooperativo) y Recompensa en Equilibrio Sostenible (Modelo Colaborativo), tal y como se indica a continuación: 


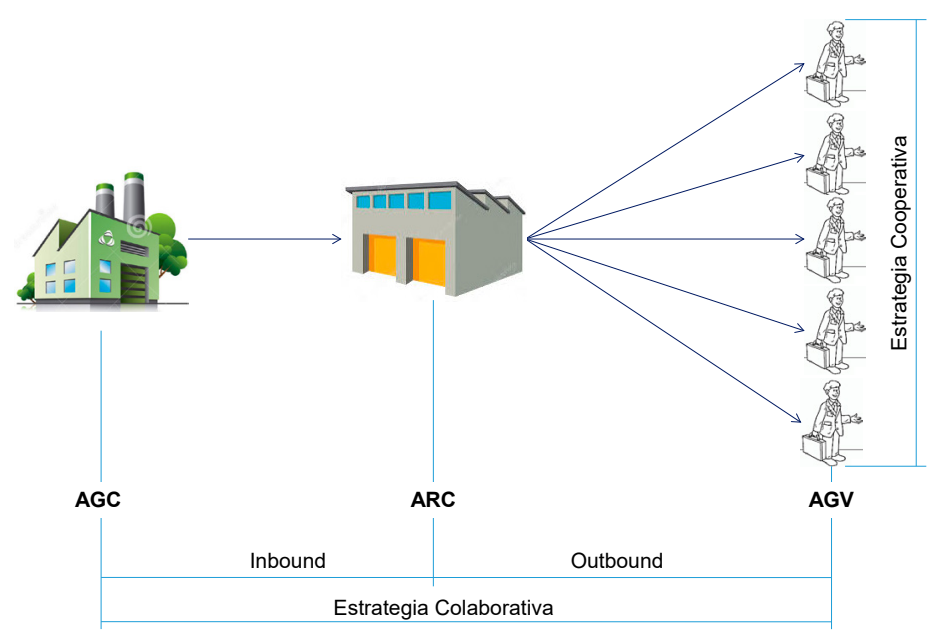

Fig. 5. Mecanismo de CVC basado en la UCE. Fuente: Autor.

\section{- UCE desde la perspectiva del AGC}

El ideal del AGC es mantener un nivel de inventarios en una cierta proporción al Ingreso Operacional Esperado, sin embargo, las particularidades del sistema productivo, la forma de gestionarlo, la gama de productos, los tamaño del lote de manufactura por gama, entre otros aspectos, han impedido el sincronismo de la UCE de Transferencia justo a tiempo entre el AGC y el ARC en la EE [4]-[30].

Motivo por el cual, el diseño de la UCE con enfoque en el AGC que se desarrolla a continuación, está basada en el Modelo para Analizar los Ciclos de Inventario de [27], con las características asociadas a las variables de decisión, que se citan a continuación [30]:

$$
\begin{gathered}
\mathrm{IO}_{\mathrm{t}}=\mathrm{IC}_{\mathrm{t}}+\mathrm{IS}_{\mathrm{t}}+\mathrm{C}_{\mathrm{W}}^{\mathrm{O}} \\
\mathrm{IC}_{\mathrm{t}}=\mathrm{p} * \mathrm{IO}_{\mathrm{t}} \\
\mathrm{IS}_{\mathrm{t}}=\mathrm{K} * \mathrm{IC}_{\mathrm{t}-1}
\end{gathered}
$$

En donde, $I O_{\mathrm{t}}$ es el ingreso operacional generado en el periodo $t, I C t$ es el inventario de consumo del periodo t, ISt es el inventario de seguridad del periodo $t, C W$ es Capital Neto no Inducido en cada periodo, $K$ es una proporción del IC durante el periodo $t$-1 y $p$ es la propensión marginal a consumir. La $C W$ está constituida no sólo por el Capital de Trabajo sino también por la Inversión en Valor de Vida, la cual, involucra bienestar en todos los aspectos para con el operario en sí [37].

Paso seguido, se reemplaza (3) en (2) y a su vez, se debe partir del supuesto que puede haber discrepancias entre el inventario planificado con el alcanzado en realidad durante el periodo $t-1$, se hace necesario reescribir esta situación en (1), procedimiento que se recoge en (4). Para facilitar, la solución del problema del ciclo de inventario del $\mathrm{AGC}$, se requiere reorganizar los términos de (4), así como efectuar la reducción en los mismos, con el objeto de perfeccionar la Ecuación en Diferencias Finitas Completa de Segundo Orden (E.D.F Completa $_{\text {) }}$, que se presenta en (5):

$$
\begin{aligned}
& \mathrm{IO}_{\mathrm{t}}=\mathrm{pIO}_{\mathrm{t}-1}+\mathrm{p}(1+\mathrm{K})\left(\mathrm{IO}_{\mathrm{t}-1}+\mathrm{IO}_{\mathrm{t}-2}+\mathrm{C}_{\mathrm{W}}^{\mathrm{O}}\right. \\
& \mathrm{IO}_{\mathrm{t}+2}-\mathrm{p}(2+\mathrm{K}) \mathrm{IO}_{\mathrm{t}+1}+\mathrm{p}(1+\mathrm{K}) \mathrm{IO}_{\mathrm{t}}=\mathrm{C}_{\mathrm{W}}^{\mathrm{O}}
\end{aligned}
$$

La E.D.F $F_{\text {Completa }}$ (5), se soluciona siguiendo el protocolo descrito en [4], que en términos generales demanda el desarrollo de las siguientes E.D.F homogéneas: i) Auxiliar; ii) Solucion General; y iii) Solucion Particular. Por medio de las cuales, se define la Solución General de la E.D.F no homogénea de forma (6):

$$
\mathrm{IO}_{\mathrm{t}}=(\mathrm{Z})^{\mathrm{t}}\left(\mathrm{C}_{1} \operatorname{Cos} \mathrm{Xt}+\mathrm{C}_{2} \operatorname{SenXt}\right)+\frac{\mathrm{C}_{\mathrm{W}}^{\mathrm{O}}}{1-\mathrm{p}}(6)
$$

En donde, las constantes $\mathrm{Z}$ y X se obtienen de la E.D.F Auxiliar Homogénea, y entre tanto, los parámetros $\mathrm{C}_{1} \mathrm{y} \mathrm{C}_{2}$ son el resultado de operar los datos históricos de entrada, suministrados por el SSD de la $\mathrm{EE}_{\mathrm{EC}}$ en cuestión. La $\mathrm{UCE}_{\mathrm{M}}$ se desagrega mediante la cuantificación del Costo Total del Inventario gestionado por el AGC $\left(\mathrm{CT}_{\mathrm{AGC}}\right)$. Tersine [35] determina el $\mathrm{CT}_{\mathrm{AGC}}$ a través de (7):

$$
\mathrm{CT}_{\mathrm{AGC}}(\mathrm{T})=\mathrm{IO}_{\mathrm{t}}+\frac{\mathrm{C}+\mathrm{nc}}{\mathrm{T}}+\frac{\mathrm{T} * \mathrm{~F} * \mathrm{IO}_{\mathrm{t}}}{2}(7)
$$


En donde, $n$ es el número total de ítems que hacen parte de la orden conjunta, $C$ es el costo conjunto de ordenar, $c$ es el costo unitario de ordenar por ítem, $T$ el intervalo de tiempo entre pedidos por Año y $F$ es la tasa de transferencia anual administra por el ARC. Haciendo uso del protocolo de optimización clásico para funciones de minimización sobre (7), se obtiene a (8):

$$
\mathrm{T}^{*}=\sqrt{\frac{2 *(C+n c)}{\mathrm{F}^{*} \mathrm{IO}_{\mathrm{t}}}}(8)
$$

En donde, $T^{*}$ representa el intervalo de tiempo óptimo entre pedidos para múltiples ítems. Tomando como base el resultado de (8) se puede establecer para cada ítem su ICt e ISt, así como también, el $\mathrm{CT}_{\mathrm{AGC}}\left(T^{*}\right)$, como se indica en (9) hasta (11):

$$
\begin{aligned}
\mathrm{IC}_{\mathrm{ij}}^{10}= & {\left[\frac{\mathrm{IO}_{\mathrm{t}} *\left(\mathrm{~T}^{*}+\mathrm{L}_{\mathrm{j}}\right)}{52 * \mathrm{P}_{\mathrm{Vi}}} * \mathrm{~W}_{\mathrm{ij}}(1-\mathrm{K})\right] } \\
\mathrm{IS}_{\mathrm{ij}}^{10}= & {\left[\left(\frac{\mathrm{IO}_{\mathrm{t}} *\left(\mathrm{~T}^{*}+\mathrm{L}_{\mathrm{j}}\right)}{52 * \mathrm{P}_{\mathrm{Vi}}}\right) * \mathrm{~W}_{\mathrm{ij}} * \mathrm{~K}\right] } \\
& \mathrm{CT}_{\mathrm{AGC}}\left(\mathrm{T}^{*}\right)=\left(1+\mathrm{F} * \mathrm{~T}^{*}\right) * \mathrm{IO}_{\mathrm{t}}
\end{aligned}
$$

En donde, $L j$ es el Lead Time negociado con cada agente $j$, Suma $\left(T^{*}+L\right)$ debe ser un entero con periodicidad en semanas, $I S_{i j}$ es el inventario de seguridad en base diez por ítem $i$ y por agente $j, P v_{i}$ es el precio de venta unitario del ítem $i, W_{i j}$ es la participación en el mercado del ítem i y por agente j, $I C_{i j}$ es el inventario de consumo en base diez por ítem $\mathrm{i}$ y por agente $j$ y $\mathrm{CT}_{\mathrm{AGC}}\left(T^{*}\right)$ representa el costo total de gestión de la $\mathrm{UCE}_{\mathrm{M}}$ por parte del AGC.

\section{- UCE desde la perspectiva del ARC}

El ARC es quien se enfrenta normalmente a los dilemas de ítem agotado o estoqueado, como reflejo del tamaño de lote de pedido pactado con el AGC [10]-[30]. Cuatrecasas-Arbós [10], sostiene que dichos dilemas afectan a ambas partes en condiciones totalmente diferenciadas, a saber: los agotados son sinónimo de lotes pequeños de producción, que exigen demasiadas horas de preparación de máquina, y con ello se creen nuevos Recursos Restrictivos de Capacidad en las instalaciones del AGC. Caso contrario ocurre con los ítems estoqueados, en donde los lotes de producción son grandes, con los cuales se puede saturar la Capacidad de Bodegaje del ARC.

Las anteriores condiciones y características identificadas, suponen el diseño de un mecanismo de CVC basado en una estrategia de integración del tipo ganar-ganar entre estos dos agentes, que balancee el Makespan del AGC con el Takt Time del ARC en un ambiente justo a tiempo, como el expresado en (11). Motivo por el cual, la $\mathrm{UCE}_{\mathrm{M}}$ se ajusta a estas necesidades, cuando se parametriza bajo el Modelo de las Dos Cajas [16], en las condiciones descritas en (12) a (13).

$$
\begin{gathered}
\mathrm{Caja}_{1}=\mathrm{IC}_{\mathrm{ij}}^{10}+\mathrm{IS}_{\mathrm{ij}}^{10} \\
\mathrm{Caja}_{2}=\mathrm{IS}_{\mathrm{ij}}^{10}
\end{gathered}
$$

En donde, las características de diseño de la caja dos, se deben establecer en un intervalo de confianza con relación al Tiempo Efectivo de Producción, para que $K$ oscile entre el cinco al quince por ciento inclusive [30], tal y como se indica en (14):

$$
\mathrm{IS}_{\mathrm{t}}=\left(\frac{\left(1-\left(\mathrm{IC}^{\text {Takt Time }}{ }_{\text {ARC }}\right)\right.}{\mathrm{IC}^{\text {Makespan }}{ }_{\text {AGC }}}\right) * 100
$$

El planeamiento de la $\mathrm{D}_{\mathrm{T}} \mathrm{Q}_{\mathrm{U}}$ bajo los lineamientos de la $\mathrm{DUM}_{\mathrm{HAS}}$, basada en el área del establecimiento comercial como limitante, con enfoque en el AGCUrbano se orienta hacia la Gestión del Bodegaje tanto Descentralizado como Centralizado, en donde se designa una Plataforma Central a una Red de Subplataformas de Distribución Zonal, que emplean vehículos de carga ecológicos para la operación, en donde la $\mathrm{UCE}_{\mathrm{M}}$ es el medio de planificación estratégica de las Capacidades Logísticas para la EEEC [13]-[25]-[38].

Conviene subrayar que la configuración física de la UCE debe estar fundamentada en el concepto Densidad de los Productos Vitales de la EE, por ser un factor determinante para el cubicaje de productos, selección de medios para el manejo y manutención de productos, dimensionamiento de edificios destinados al bodegaje de productos y selección de la flotilla de vehículos de carga. Concepto relacionado con los criterios fundamentales para el diseño del embalaje de un producto, a saber: peso y/o volumen [1]-[30]. Por lo demás, el criterio a ser seleccionado para el producto en cuestión, será aquel que sea el más representativo entre los dos, que para esta investigación será un Inbox de capacidad máxima para 25 kilos por litro, medida estándar para la UCE en estudio [30].

El ARC permite mitigar la ambigüedad en la gestión de la $\mathrm{UCE}_{\mathrm{M}}$ con respecto a los deseos del $\mathrm{AGV}$, a la vez, que reduce la incertidumbre y mejora la prospectiva de la $\mathrm{D}_{\mathrm{T}} \mathrm{Q}_{\mathrm{U}}$ puede hacer uso de la estrategia Postergación de la Gestión del Sistema de DUM HAS $_{\text {S }}$ [30]. El concepto postergación está relacionado en la DUM $_{\text {HAS }}$ con: i) Mitigar la variabilidad de la $\mathrm{D}_{\mathrm{T}} \mathrm{Q}_{\mathrm{U}}$; ii) Reducir el nivel del inventario en la EE; y iii) Mejorar los niveles de servicio aguas arriba de la EE. Para cumplir con estos propósitos, se debe consolidar la $\mathrm{UCE}_{\mathrm{M}}$ en atención a la distancia, tiempo 
de viaje, zonas de consumo georreferenciadas, entre otros criterios [38].

La consolidación de la $\mathrm{UCE}_{\mathrm{M}}$ en la $\mathrm{EE}_{\mathrm{EC}}$, se puede obtener, por medio de la utilización de la técnica Regla de la Raíz Cuadrada bajo el supuesto de equiprobabilidad del inventario en todas las instalaciones industriales (15):

$$
\mathrm{I}_{2}=\mathrm{I}_{1} *\left(\sqrt{\frac{\mathrm{n}_{2}}{\mathrm{n}_{1}}}\right)
$$

En donde, $I_{1}$ es la $\mathrm{UCE}_{\mathrm{M}}$ gestionada por la DUM $_{\text {HAS }}$ con $n 1$ ubicaciones, $I_{2}$ es la UCE $\mathrm{UCE}_{\mathrm{M}}$ gestionada por la DUM $\mathrm{DUS}_{\mathrm{HAS}}$ con $n 2$ localizaciones, $n 1$ número actual de localizaciones que conforman la $\mathrm{DUM}_{\mathrm{HAS}}$ y $n 2$ número de localizaciones nuevas

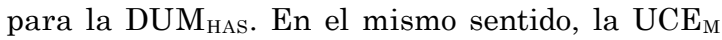
para la $\mathrm{EE}_{\mathrm{EC}}$ consolidada, se puede obtener con la técnica Regla de la Raíz Cuadrada con soporte en la Demanda Agregada de todas las instalaciones industriales (16):

$$
\mathrm{UCE}(\%)=\left(\sum_{i=1}^{n}\left(\sqrt{\frac{d_{i}}{\mathrm{D}_{\mathrm{T}}}}\right)-1\right) * 100
$$

En donde, $D_{T}$ es la demanda agregada de la $\mathrm{EE}_{\mathrm{EC}}$ y $d_{i}$ es la demanda georreferenciada en la $\mathrm{EE}_{\mathrm{EC}}$. Ahora bien, en la DUM $\mathrm{HAS}_{\mathrm{S}}$ con enfoque en el AGV, la etapa Inbound se acopla con la etapa Outbound mediante el mecanismo de CVC denominado UCE, el cual, está basado en el criterio de Recompensa en Equilibrio Sostenible, formulación que se presente en (17):

$$
\mathrm{CT}_{\mathrm{EE}_{\mathrm{EC}}}=\operatorname{Min}\left\{\frac{1}{2}\left[\frac{\mathrm{UC}_{\mathrm{ARC}}^{10}}{\mathrm{UCE}_{\mathrm{AGV}}^{10}}+\frac{\mathrm{UCE}_{\mathrm{AGV}}^{10}}{\mathrm{UC}_{\mathrm{ARC}}^{10}}\right]\right\} \mathrm{CT}\left(\mathrm{T}^{*}\right)
$$

En donde, $\mathrm{CT}_{\mathrm{EEEC}}$ es el costo total de la estrategia de integración ganar-ganar para la $\mathrm{UCE}_{\mathrm{M}}$.

\section{- UCE desde la perspectiva del AGV}

La integración vertical en la $\mathrm{EE}_{\mathrm{EC}}$ está constituida por una estrategia ganar-ganar de cooperación, que tiene por objetivo CVC en forma sostenible, para lo cual, los agentes se comparten información, asumen costos conjuntos, están dispuestos a negociar un acuerdo antes del flujo de pagos laterales y ante todo se colaboran, al mismo tiempo, que el Contrato Contractual y Vinculante garantiza a las partes, su independencia económica, autonomía administrativa, y respecto por su cultural e idiosincrasia organizacional [30]. Situación que se modela en el campo de la investigación de operaciones estocásticas, a través, de la Teoría de Juegos de Trasferencia de Utilidad (Juegos TU) [18].
Los Juegos TU se ocupan del estudio de la cooperación entre agentes, situación que promueve la formación de una Coalición estable que perdure en el tiempo, para lo cual, se debe modelar bajo la disciplina matemática del Óptimo Social, a fin de analizar su Distribución de Beneficios, en especial el de tipo económico, lo que implica evaluarla bajo el marco axiomático de Shapley, los conceptos de solución de núcleo y del estudio de las propiedades de valor, que puede alcanzar la Integración Vertical de la EE en forma ecuánime, cuando sus agentes mejoran la coordinación de las estrategias, que la constituyen como tal [3]-[17]-[18].

Estos planteamientos, permiten suponer que la integración vertical de la EE vía gestión de inventarios conjuntos con enfoque en el AGV, se logra mediante la centralización de órdenes mutuas de pedido, las cuales permiten el planeamiento de la $\mathrm{D}_{\mathrm{T}} \mathrm{Q}_{\mathrm{U}}$ con lineamientos en la DUM $\mathrm{DAS}_{\mathrm{HAS}}$, basada en el área del establecimiento comercial como limitante, haciendo uso de las estrategias OPT y OPA tal como se ilustra en la Fig. 6, que en sí, son una innovación en la entrega capilar [24]. Por tanto, el objetivo en este punto del estudio se reduce al reparto de los gastos asociados con la realización conjunta de órdenes de pedido, por parte del AGV.

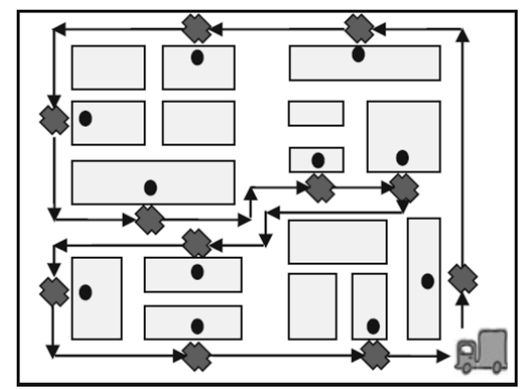

a) Estrategia OPT

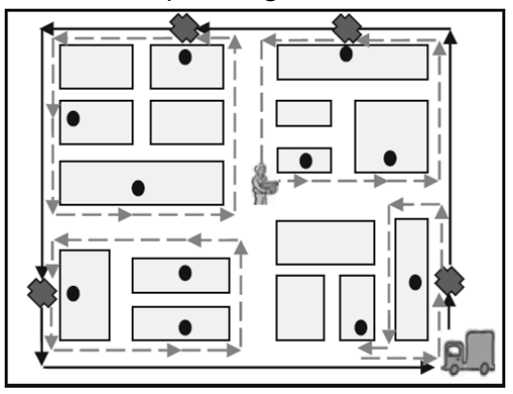

b) Estrategia OPA

Fig. 6. Estrategias usadas en la entrega capilar. Fuente: [24].

Los AGV de manera general, usan el sistema de cantidad económica de pedido (CEP), para gestionar su inventario de consumo, que en los Juegos TU, es una situación multi-agente donde cada uno de los agentes que conforman la coalición se enfrentan a un problema de revisión continúa, en el cual, el Sis- 
tema de Inventario y Transporte, permite modelar las necesidades del AGV en que el Modelo Básico Sin Costo de Bodegaje, con Bodegaje Capacitado y con Costo de Transporte, es uno de los casos de estudio que puede ser implementados en los procesos de integración de agentes bajo los lineamientos de la $\mathrm{DUM}_{\mathrm{HAS}}$ [15]. Además, este modelo básico de CEP no permite escases, a la vez, que la orden de pedido está valuada en términos del costo semifijo asociado al transporte que depende de la distancia, que existe entre el $A G V_{i}$ al ARC [14].

En cuanto a la gran coalición, considere $\mathrm{N}:=\{1$, ....., $n\}$, se define los parámetros asociados a cada i $\in N$, como: $a>0$, el coste fijo por pedido; $a_{i}>0$, el costo de transporte por pedido; $d_{i}>0$, la $\mathrm{UCE}_{\mathrm{M}}$ por unidad de tiempo; y $K_{i}>0$, la capacidad de bodegaje del $\mathrm{AGV}_{\mathrm{i}}$. En términos general, la cooperación en este modelo consiste en constituir una coalición $\mathrm{S} \subseteq N$ para realizar pedidos conjuntos en procura de racionalizar sus costos c(S), en virtud de las siguientes dos suposiciones [15]: i) Todos los $\mathrm{AGV}_{\mathrm{i}}$ están ubicados en la misma ruta de entrega: si un grupo de agentes realiza una orden conjunta $S \subseteq N$, el costo fijo es la suma de una componente $a$ y otra equivalente a la distancia maximal entre $A G V_{i}$ al ARC; y ii) Si se forma una coalición de órdenes de pedido S, el ARC asigna a cada una de ellas un costo igual a: $a+\operatorname{Max}_{i \in s}\left\{a_{i}\right\}$. Es necesario recalcar que el modelo se denota por la tupla $\left(N, a,\left\{a_{i}\right\}_{\mathrm{i} \in \mathrm{N}}\right.$, $\left.\left\{\mathrm{d}_{\mathrm{i}}\right\}_{\mathrm{i} \in \mathrm{N}},\left\{\mathrm{K}_{\mathrm{i}}\right\}_{\mathrm{i \in N}}\right)$.

Según Fiestras-Janeiro et al. [15], la política óptima para la gestión del inventario individual de este modelo se puede expresar por la función de costo total dada en (18), entre tanto, la cooperación implica que las ordenes de pedido sean coordinadas, de tal forma, que las longitud de ciclo tanto individual como conjunta deben ser coincidentes bajo las dos suposiciones citadas en el párrafo anterior, para cada coalición no vacía $\mathrm{S} \subseteq \mathrm{N}$, cuyo óptimo social en que se puede suscribir el acuerdo contractual vinculante entre los $\mathrm{AGV}_{\mathrm{i}}$ con el ARC queda definido por (19):

$$
\begin{gathered}
\mathrm{C}_{\mathrm{i}}:=\frac{\text { Costo de un Ciclo }}{\text { Longitud del Ciclo }}=\left(\mathrm{a}+\mathrm{a}_{\mathrm{i}}\right) \frac{\mathrm{d}_{\mathrm{i}}}{\mathrm{K}_{\mathrm{i}}} \\
\mathrm{C}_{\mathrm{s}}:=\frac{\text { Costo de un Ciclo }}{\text { Longitud del Ciclo }}=\frac{\max _{\mathrm{ies}}\left\{\mathrm{a}+\mathrm{a}_{\mathrm{i}}\right\}}{\min _{\mathrm{j} \in \mathrm{s}}\left\{\mathrm{K}_{\mathrm{j}} / \mathrm{d}_{\mathrm{j}}\right\}}
\end{gathered}
$$

Conocida la política óptima que promueve este modelo de gestión del inventario conjunto entre los AGVi con el ARC, a sabiendas que la misma posee las condiciones para las cuales es benéfica la cooperación desde el criterio del óptimo social incluida la subaditividad [14], se da paso al estudio de cómo repartir el óptimo social alcanzado entre los agentes que generan la orden de pedido conjunta, para lo cual, se adopta como mecanismo de asignación de costos La Regla de las Dos Líneas $\left(\mathrm{R}_{2 \mathrm{~L}}\right)$ propuesta por [15].

La $R_{2 L}$ se fundamenta en los siguientes dos conjuntos de permutaciones [15]: i) $\mathrm{P}_{1}$ : El conjunto está formado por las permutaciones que restituyen el orden dado por los costos de transporte, es decir, se elegirán aquellos conjuntos en donde $a_{i} \geq a_{j}$ y donde el marginal (i) $\leq$ marginal (j); y ii) $\mathrm{P}_{2}$ : El conjunto de permutaciones lo constituyen aquellas que restablecen el orden dado por las razones obtenidas de la division entre la demanda y la capacidad de bodegaje, en otras palabras, se elegirán aquellos conjuntos en donde $d_{i} / K_{i} \geq d_{j} / K_{j}$ y donde el marginal (i) $\leq$ marginal (j).

La $R_{2 L}$ establece las condiciones del Juego $\mathrm{Tu}$ bajo las cuales los repartos del Óptimo Social están en su núcleo, cuya expresión matemática se recoge en (20):

$$
\mathrm{R}_{2 \mathrm{~L}}:=\frac{1}{2\left|\mathrm{P}_{1}\right|} \sum \mathrm{M}_{\mathrm{g}} \mathrm{P}_{1}+\frac{1}{2\left|\mathrm{P}_{2}\right|} \sum \mathrm{M}_{\mathrm{g}} \mathrm{P}_{2}
$$

\section{Evaluación técnico-económica de UCE en EE}

Una empresa, es la sumatoria exitosa de la ejecución de proyectos de inversión. Siendo una de las técnicas más empleadas en el Análisis de Inversiones el Valor Presente Neto (VPN), para medir la Creación de Valor Sostenible que se espera de la ejecución del Ciclo de Inversión y Financiamiento (CIF) de un Proyecto de Inversión. En donde, la herramienta que permite valorar el impacto del VPN en función del CIF es el Flujo de Caja Totalmente Neto (FCTN), el cual, se construye con información parcial proveniente del contexto en que se origina el proyecto de inversión [31], por lo general, situación que amerita el uso de la teoría de los Subconjuntos Borrosos [23], que mitigue el margen de tomar una decisión que destruya valor entorno a una alternativa de inversión [31].

Lo dicho hasta aquí supone que, la configuración de una $E E_{E C}$ no es indiferente frente a esta realidad, no sólo por los procesos de negociación que afronta su coalición de agentes sino también por su propia concepción fáctica [23]-[31]. En otras palabras, el implementar una estrategia de integración del tipo ganar-ganar, por medio de un contrato que es tanto contractual como vinculante entre el ARC con los AGVi, deberá estar concebida en un ambiente de incertidumbre y ambigüedad, con el objeto de hacerla flexible y dinámica en favor de su sostenibilidad durante su vigencia [6]-[23]-[31]-[39].

Por tanto, esta etapa tiene la finalidad de perfeccionar el procedimiento sistemático de diseño de la EEEC desde el enfoque del AGV hasta aquí descrito, razón por la cual, la UCE debe ser evaluada no sólo técnicamente sino también económicamente, por ser un mecanismo de CVC diseñado 
en condiciones de incertidumbre e ambigüedad, se necesita que el SSD este delineado con el paradigma holónico, por un lado, y soportado en Conjuntos Borrosos, por el otro.

Los conjuntos borrosos son de naturaleza no estadística y emulan el razonamiento humano, a quienes se les asocia una función de pertenencia [39]. Por otra parte mejora el SSD frente al proceso de estimar el riesgo que experimenta el FCTN, al pasar de un esquema de asignación de una función de distribución de probabilidad a incorporar una aproximación posibilistica en el análisis del CIF [6], que para la presente investigación, está referido al punto de equilibrio de la $\mathrm{UCE}_{\mathrm{M}}$ entre la Inbound y la Outbound de la $\mathrm{EE}_{\mathrm{EC}}$ con enfoque en el AGV.

Con respecto al perfeccionamiento del mecanismo de CVC en condiciones de incertidumbre e ambigüedad, es necesario modificar en términos matemáticos el FCTN, mediante el método del equivalente de certeza con ajuste en la prima libre de riesgo [31], situación que puede ser expresada con (21):

$$
\mathrm{FCTN}_{\mathrm{t}}^{*}=\mathrm{CRt}_{\mathrm{Rt}} * \mathrm{FCTN}_{\mathrm{t}}
$$

En donde, FCTN* es el FCTN cierto para el periodo $t, \mathrm{FCTN}_{t}$ es el FCTN que incluye un cierto nivel de riesgo para el periodo $t, r K$ costo de oportunidad (Rentabilidad Libre de Riesgo) exigido a la integración de la $\mathrm{EE}_{\mathrm{EC}}$, PLR Prima Libre de Riesgo a ser aplicada durante la vigencia del contrato contractual y vinculante entre los agentes de la $\mathrm{EE}_{\mathrm{EC}}$, su inclusión en el $\mathrm{FCTN}_{\mathrm{t}}$ se indica en (22) y $C R$ hace referencia al coeficiente reductor para el periodo $t$, valor que oscila entre cero y uno.

$$
\mathrm{FCTNt}=\frac{\left(1+\mathrm{r}_{\mathrm{K}}\right)^{\mathrm{t}}}{1+\mathrm{r}_{\mathrm{K}}+\mathrm{P}_{\mathrm{LR}}} \quad * \mathrm{FCTN}_{\mathrm{t}}
$$

El VPN en condiciones de incertidumbre e ambigüedad $\left(\mathrm{VPN}_{\mathrm{AIA}}\right)$, se puede calcular mediante la aplicación de los números borrosos [23], los cuales, permiten parametrizar uno o más criterios inherentes al FCTN para cada uno de los t periodos que comprenden la integración, que se hará mediante el empleo de los Números Borrosos Triangulares (NBT) con Escala Lexicográfica Binaria (a-Corte), dados los patrones (Fig. 7) e intervalo de confianza (23):

$$
a_{\text {Corte }}=\left[a_{t}+\alpha\left(m_{t}-a_{t}\right), b t-a\left(b_{t}-m_{t}\right)\right]
$$

En donde, $a_{t}$ es el valor pesimista a lograr en el periodo $t, m_{t}$ es el valor más probable a lograr en el periodo $t, b_{t}$ es el valor optimista a lograr en el periodo $t \mathrm{y}$ a valor asociado a un nivel de pertenencia dentro del intervalo [0,1] de los NBT. La expresión (24) permite cuantificar el $\mathrm{VPN}_{\mathrm{AIA}}$ :

$$
\operatorname{VPN}_{\mathrm{IA}}^{\mathrm{A}}=\left(\mathrm{I}_{0}\right)+\theta_{\mathrm{t}}^{\mathrm{a}} \sum_{i=1}^{n}\left[\frac{\mathrm{FCTN}_{\mathrm{t}}^{\mathrm{LI} a}}{\left(1+\mathrm{r}_{\mathrm{LI}}^{\mathrm{a}}\right)^{\mathrm{t}}} \cdot \frac{\mathrm{FCTN}_{\mathrm{t}}^{\mathrm{LS} a}}{\left(1+\mathrm{r}_{\mathrm{LS}}^{\mathrm{a}}\right)^{\mathrm{t}}}\right]
$$

En donde, $I_{O}$ es la inversión inicial, $L I_{\alpha}$ límite inferior del FCTN dado el nivel de a-corte, $L S_{a}$ límite superior del FCTN dado el nivel de a-corte, $\theta^{a}$ coeficiente reductor dado el nivel de a-corte y $r^{a}$ costo de oportunidad dado el nivel de a-corte para el periodo $t$. Esta última expresión permite calcular posibles valores de CVC entre los cuales, para cada nivel de confianza, se confía establecer su magnitud real, por medio de la cual, se pueda viabilizar para el mediano y largo plazo, el diseño de la EEEC en función de la UCE con enfoque en el AGV.

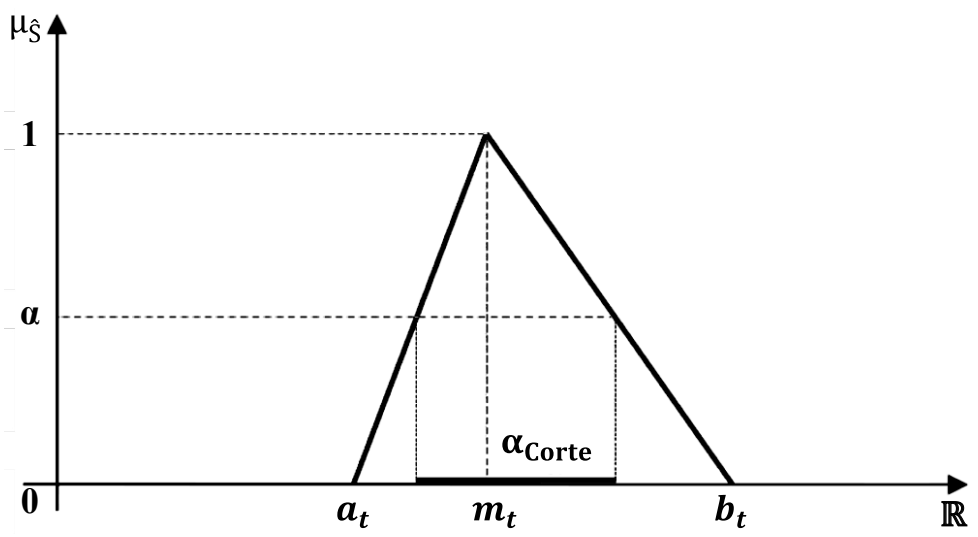

Fig. 7. Distribución PERT de una a-corte con NBT. Fuente: Autor. 


\section{Resultados}

Para mostrar la importancia del trabajo expuesto, se tomó como referente una compañía líder del sector de autopartes de Colombia con sede en la ciudad de Bogotá, siguiendo los pasos descritos en el ítem anterior, los cuales, se presenta a continuación:

\section{A. Información requerida por $M I E L_{C V C}$}

Una vez, surtida la aplicación del Método SCAMPER, la información base requerida por el diseño estratégico de la UCE con respecto al AGV en condiciones de vaguedad e incertidumbre, se cita formalmente, a continuación:

La familia de productos para el estudio, está conformada por las referencias: 50113, 50129 y 50133, que poseen una participación del $7.21 \%$ del IO (Analisis de Pareto), que representa 62,980 USD para el año base. En contraste con el año anterior, en el que alcanzaron los 75,220 USD, por otra parte, p se estimó en $50 \%$ anual (Analisis Estadístico), a pesar de que la $\mathrm{EE}_{\mathrm{EC}}$ como tal, viene destruyendo valor a una tasa del 3.34\% anual. Además, el AGC gestiona un IS a una tasa del $28 \%$ anual, así como también, administra un CW a una tasa del $60 \%$ anual, en la cual, se incluye la inversión de valor de vida relacionada con el trabajador y su familia, con el cual, se proyecta un crecimiento en su mercado metal del $4,5 \%$ anual (Analisis Microeconómico).

Otro rasgo, obtenido a partir del Análisis de Pareto, tiene que ver con la participación en los IO proyectados para el primer año, por parte de las referencias 50133, 50113 y 50129, que sigue la distribución del $52 \%, 29 \%$ y $19 \%$ respectivamente. Así mismo, los parámetros a tenerse en cuenta por parte del AGC en el modelado de la UCEM adicionales son: i) La DUMHAS está conformada por tres $\mathrm{AGVs}$, sistema que le cuesta al ARC en promedio el $35 \%$ anual sobre Inbox; ii) $\mathrm{F}=48 \%$ anual; v) Costo de hacer una orden de pedido $=23,850 \mathrm{COP} /$ Orden; vi) Costo de emitir una corrida de producción $=32,410 \mathrm{COP} /$ Solicitud; $\mathrm{y}$ vii) Costo promedio del servicio de bodegaje $=1,560$ COP/Inbox-Año.

Por otro lado, el ARC configuro la UCEM para unitarizar en embalaje homogéneo sus productos manufacturados en un SKU con capacidad máxima de 25 kilos/Inbox, cubicaje que representa un IO promedio de 120,000 COP/Inbox. Así mismo, estimo los precios unitarios de venta al público en COP del 50133, 50113 y 50129 para el primer año en 4,900; 4,500 y 3,600 respectivamente. Con respecto a, la unidad de empaque para las referencias 50133, 50113 y 50129, son de Una Unidad, Cuatro Unidades y Una Unidad respectivamente.

Hay que mencionar, además que el ARC deberá hacer adecuaciones en layout por valor de 752,000 USD están representadas en elementos de manutención de inventarios. Además, se requiere acondicionar el edificio industrial con un sistema inmótico por valor de 173,000 USD, inversión que brindara calidad de vida a sus colaboradores. Más aun, la mejora en el layout proporciona 6,480 Inbox de bodegaje a la $\mathrm{EE}_{\mathrm{EC}}$, la cual, será ejecutada en dos etapas. En la fase uno, se instalaran 3,360 posiciones de Inbox, siguiendo el CIF que a continuación se cita: i) $30 \%$ con Recursos Propios; ii) $\mathrm{P}_{\mathrm{LR}}=3 \%$ efectiva anual; iii) $r_{K}=10 \%$ efectiva anual; iv) Tasa Social de Descuento $=12 \%$ efectiva anual (Colombia); y v) Costo de financiamiento máximo $=24 \%$ efectivo anual. En la fase dos, se instalan las restantes posiciones un lustro después.

Habría que decir también, que el mecanismo de CVC en que se soporta la estrategia de integración del tipo ganar-ganar en función de la $\mathrm{UCE}_{\mathrm{M}}$ en Condiciones de vaguedad e Incertidumbre, en términos de la evaluación financiera posee las siguientes características: i) La escala semántica es Pentaria; ii) El expertometro definió el límite superior con factor de 1,05 sobre el FCTN*; y iii) La $r_{\mathrm{K}}$ es constante durante la vigencia de la integración de la $\mathrm{EE}_{\mathrm{EC}}$.

Finalmente, los AGVs se incorporan al sistema DUM $_{\text {HAS }}$ con los costos de transporte que se indican en la tabla 1.

Tabla 1. Costos Logísticos Gestionados POR LOS AGVS EN EL AÑo 1

\begin{tabular}{|c|c|c|c|}
\hline AGV & $\begin{array}{c}\mathrm{L}^{*} \\
\text { (Semana) }\end{array}$ & $\begin{array}{c}\mathrm{c} \\
(\mathrm{COP} / \text { Inbox-Año) }\end{array}$ & $\begin{array}{c}\mathrm{a}_{\mathrm{i}} \\
(\mathrm{COP} / \text { Envio })\end{array}$ \\
\hline Institucional & 1 & $\$ 1.856$ & $\$ 20.825$ \\
\hline Local & 1 & $\$ 2.174$ & $\$ 17.500$ \\
\hline Regional & 1 & $\$ 2.225$ & $\$ 14.370$ \\
\hline
\end{tabular}

Fuente: Autor.

El costo variable de transporte presentado en la tabla 1, está referido a un Inbox con capacidad máxima de 25 kilos, mientras que $a=2,760,000$ COP. Costo que está referido a un envió urgente de mínimo de 23 Inbox en atención a la (16) a favor de los AGVs.

\section{B. UCE desde la perspectiva del AGC}

Operando (1) a (11) con la información suministrada se obtiene la propuesta de gestión de inventarios del AGC, a partir de los tiempos de entrega requeridos por el ARC. En la tabla 2, se recoge la propuesta, como tal, con un Costo Total para el primer año de 233,396,590 COP/Año.

Tabla 2. Uce desde la Perspectiva del AGC en el AÑo 1

\begin{tabular}{|c|c|c|c|c|}
\hline \multirow{2}{*}{ PRODUCTO } & \multirow{2}{*}{$\mathrm{T}^{*}$} & \multirow{2}{*}{$\mathrm{L}^{*}$} & \multicolumn{2}{|c|}{ (Inbox/Pedido) } \\
\cline { 4 - 5 } & (Semana) & (Semana) & $\mathrm{IC}_{\text {AGC }}$ & IS $_{\text {AGC }}$ \\
\hline 50133 & 1 & 2 & 39 & 12 \\
\hline 50113 & 1 & 3 & 32 & 9 \\
\hline 50129 & 1 & 4 & 24 & 7 \\
\hline
\end{tabular}

Fuente: Autor. 


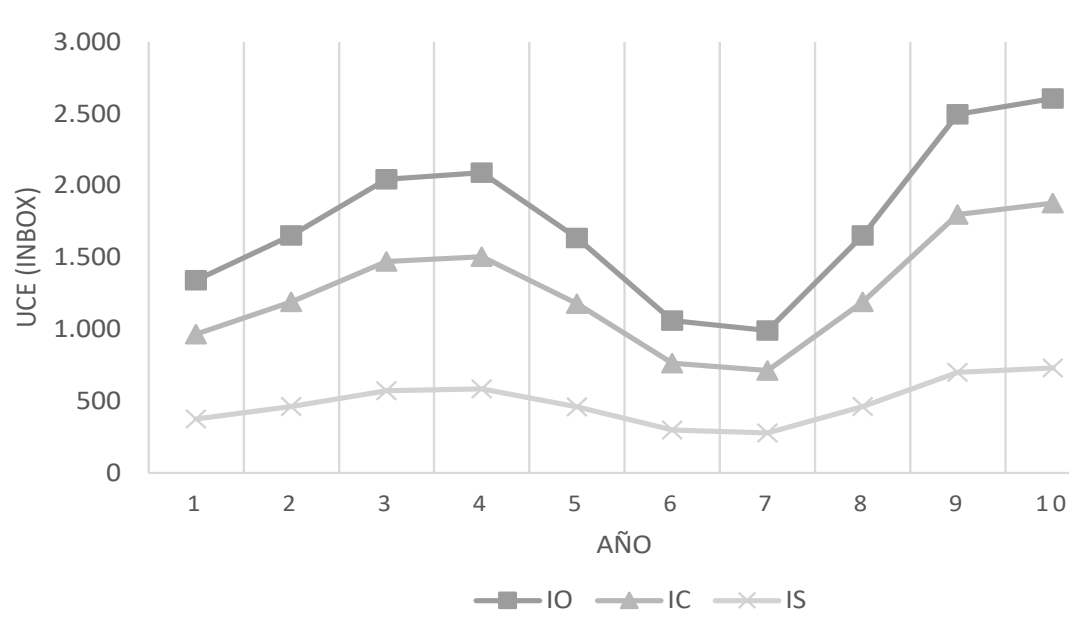

Fig. 8. Distribución de la UCE en el AGC.

Fuente: Autor.

Se debe agregar que de seguir la tendencia indicada en la Fig. 7, la $\mathrm{EE}_{\mathrm{EC}}$ deberá implementar una estrategia de innovación antes del año 4, con el propósito de evitar una pérdida de valor compartido en los próximos tres años.

\section{UCE desde la perspectiva del ARC}

La tabla 3, recoge la distribución de la demanda del año 1 con respecto a los AGVs, misma que sirvió de base para construir la tabla 2 , y a su vez, es el mismo dato agregado que se presenta en (8). Más aun, la distribución presentada en la tabla 3 , fue construida con base al análisis estadístico realizado a los IO netos por familias de productos, del cual, se pudo establecer para la presente investigación, que las referencias 50113, 50129 y 50133, son los productos líder en la actualidad.

Tabla 3. Distribución de la Demanda del Año 1

\begin{tabular}{|c|c|c|c|c|}
\hline \multirow{2}{*}{ AGV } & \multicolumn{3}{|c|}{ (Inbox/Año) } & \multirow{2}{*}{$\begin{array}{c}\text { DEMANDA } \\
\text { (Inbox/Año) }\end{array}$} \\
\cline { 2 - 4 } & 50133 & 50113 & 50129 & 241 \\
\hline Institucional & 61 & 32 & 148 & 333 \\
\hline Local & 187 & 96 & 50 & 771 \\
\hline Regional & 434 & 287 & 50 & \\
\hline
\end{tabular}

Fuente: Autor.

El manejo matemático de (12) a (15) permite establecer el ajuste a la gestión del inventario por parte del ARC, con respecto a la propuesta planteada por el AGC. En la tabla 4, se presentan estos ajustes con respecto tanto a los AGVs como a las referencias en estudio.
Tabla 4. Uce desde la Perspectiva del ARC en el AÑo 1

\begin{tabular}{|c|c|c|c|c|}
\hline \multirow{2}{*}{ AGV } & \multicolumn{3}{|c|}{ (Inbox/Pedido) } & \multirow{2}{*}{$\begin{array}{c}\text { IC }_{\text {AGV }} \\
\text { (Inbox) }\end{array}$} \\
\cline { 2 - 4 } & 50133 & 50113 & 50129 & \\
\hline I & 4 & 3 & 15 & 40 \\
\hline L & 11 & 8 & 5 & 24 \\
\hline R & 25 & 23 & 5 & 53 \\
\hline Caja 1 & 40 & 34 & 25 & (Inbox) \\
\hline Caja 2 & 46 & 39 & 28 & (Inbox) \\
\hline \multicolumn{5}{|c|}{ Fuente: Autor. } \\
\hline
\end{tabular}

De la tabla 4, se puede evidenciar que el IS $_{\text {ARC }}$ está dentro de los parámetros establecidos por [30], el cual, se obtiene de la diferencia entre la caja 2 y la caja 1 , que en comparación con el IS $_{\text {AGC }}$, está ocho puntos por debajo en promedio en todos las referencias, como consecuencia de la centralización del bodegaje.

\section{UCE desde la perspectiva del AGV}

La tabla 5, muestra los parámetros correspondientes al inventario de consumo y la capacidad de bodegaje de cada uno de los AGVs, para quienes la presente investigación va evaluar la posibilidad de que hagan parte de la integración vertical. Asimismo, en la tabla 6 se presenta la aplicación de (18) a (19) por medio de las cuales, se logra establecer para cada coalición su Óptimo Social Global, mientras que aplicar la (20) implica hacer la distribución entre los AGVs que cooperaran, y para quienes se hace necesario el diseño del Acuerdo Contractual y Vinculante, en donde, la tabla 7, da a conocer el uso de las reglas de asignación para el $R_{2 \mathrm{~L}}$, mientas que la tabla 8 , condensa la distribución bajo dos perspectivas de mejora, desde el Óptimo Social. 
Tabla 5.Información Correspondiente al AÑo 1 de los AgV

\begin{tabular}{|c|c|c|c|}
\hline AGV & di (Inbox/Quincena) & Ki (Inbox) & di/Ki(Quincena $\left.{ }^{-1}\right)$ \\
\hline I & 40 & 74 & 0,54 \\
\hline L & 48 & 83 & 0,58 \\
\hline R & 54 & 90 & 0,60 \\
\hline
\end{tabular}

Fuente: Autor.

Tabla 6. Estudio de Posibles Coaliciones para el año 1 entre los AGV

\begin{tabular}{|c|c|c|c|c|}
\hline Coalición & 0 & $\{\mathrm{I}\}$ & $\{\mathrm{L}\}$ & $\{\mathrm{R}\}$ \\
\hline $\mathrm{Ca}(\mathrm{COP})$ & $\$ 0$ & $\$ 1,942.162$ & $\$ 2,081.928$ & $\$ 2,121.600$ \\
\hline
\end{tabular}

\begin{tabular}{|c|c|c|c|c|}
\hline Coalición & $\{\mathrm{I}, \mathrm{L}\}$ & $\{\mathrm{I}, \mathrm{R}\}$ & $\{\mathrm{L}, \mathrm{R}\}$ & $\mathrm{N}$ \\
\hline $\mathrm{Ca}(\mathrm{COP})$ & $\$ 2,081.928$ & $\$ 2,155.800$ & $\$ 2,160.000$ & $\$ 2,160.000$ \\
\hline
\end{tabular}

Tabla 7. Uso de las Reglas de Decisión para el AÑo 1 entre los AGV

\begin{tabular}{|c|c|c|c|r|}
\hline Regla & DOMINANCIA & I & L & \multicolumn{1}{c|}{ R } \\
\hline $\mathrm{a}(\mathrm{i})>=\mathrm{a}(\mathrm{j})$ & $(\mathrm{L}, \mathrm{I}, \mathrm{R})$ & $\$ 0$ & $\$ 2.120 .328$ & $\$ 0$ \\
\hline$(\mathrm{di} / \mathrm{Ki})>=(\mathrm{dj} / \mathrm{Kj})$ & $(\mathrm{R}, \mathrm{L}, \mathrm{I})$ & $\$ 0$ & $\$ 0$ & $\$ 2,199.672$ \\
\hline
\end{tabular}

Fuente: Autor.

Tabla 8. Óptimo Social a distribuir en el AÑo 1 entre los AGV

\begin{tabular}{|c|r|r|r|c|}
\hline Agente & $\mathrm{I}$ & $\mathrm{L}$ & $\mathrm{R}$ & $\begin{array}{c}\text { Óptimo } \\
\text { Social }\end{array}$ \\
\hline Óptimo ( ) & $\$ 653.087$ & $\$ 725.070$ & $\$ 781.842$ & $\$ 2,160.000$ \\
\hline $\mathrm{TL}(\mathrm{N}, \mathrm{c})$ & $\$ 0$ & $\$ 1,060.164$ & $\$ 1,099.836$ & $\$ 2,160.000$ \\
\hline \multicolumn{5}{|c|}{ Fuente: Autor. }
\end{tabular}

Tabla 9. Distribución de la UCE m $_{\text {pactada para el AÑo } 1}$

\begin{tabular}{|c|c|c|c|c|}
\hline \multirow{2}{*}{ AGV } & \multicolumn{3}{|c|}{ (Inbox/Pedido) } & \multirow{2}{*}{$\begin{array}{c}\text { ICAGV } \\
\text { (Inbox) }\end{array}$} \\
\cline { 2 - 4 } & 50133 & 50113 & 50129 & 40 \\
\hline I & 8 & 7 & 25 & 48 \\
\hline L & 21 & 18 & 9 & 54 \\
\hline R & 24 & 21 & 9 & 43 \\
\hline Caja 1 & 53 & 46 & (Inbox) \\
\hline Caja 2 & 64 & 55 & 52 & (Inbox) \\
\hline
\end{tabular}

Fuente: Autor.

En la tabla anterior, se presenta el valor de Shapley de (N, c) bajo el nombre de Óptimo (C [14]-[17]. La propuesta del valor de Shapley está en el núcleo del juego TU de costo (N, c) definido en [15]-[18]. Considerando el reparto de costos entre los agentes I y L, bajo (25):

$$
\text { Óptimo (I) + Óptimo (L) } \leq \mathrm{Cs}\{\mathrm{I}, \mathrm{L}\}
$$

Motivo por el cual, se justifica que Óptimo (C pertenece a $\mathrm{C}_{\mathrm{s}}$, y por ende, en este caso, en particular, se cuenta con dos estructuras de asignación de Óptimo Social. Así que, la Recompensa en Equilibrio Sostenible que concede los AGVs a la Inbound de la $\mathrm{EE}_{\mathrm{EC}}$, como consecuencia de su propuesta de pedidos es del $6.84 \%$ anual, respuesta que se obtiene de aplicar (17), y a su vez, se expone en la tabla 9 . 


\section{E. Evaluación Técnica-Financiera de la UCE}

La evaluación técnico-financiera con la aplicación de la lógica difusa, cuyo objetivo es perfeccionar la estrategia de integración del tipo ganar-ganar que permita que la $\mathrm{EE}_{\mathrm{EC}}$ pueda establecer la $\mathrm{UCE}_{\mathrm{M}}$, por medio de instrumentos de estimación de CVC, cuya finalidad es sacar provecho del riesgo e incrementar los índices de rentabilidad, entre los agentes que la conforman. Se realiza el análisis sobre los criterios de evaluación financiera de proyectos, tales como: $\mathrm{VPN}_{\mathrm{AIA}}$ y la tasa verdadera de rentabilidad en condiciones de incertidumbre e ambigüedad ( $T V R_{\mathrm{AIA}}$ ); mediante el uso de los números borrosos triangulares, que permita la toma de decisiones, con la cual, se pueda aproximar para medir la rentabilidad y la viabilidad del CIF en pesos constantes [30]-[31].

La tabla 10, presenta distintos niveles de presunción bajo los cuales se puede establecer, el VPN $\mathrm{VII}_{\mathrm{A}} \mathrm{y}$ $\mathrm{TVR}_{\mathrm{AIA}}$ que permiten la toma de decisiones frente a la $\mathrm{CVC}$, para los agentes que conforman la $\mathrm{EE}_{\mathrm{EC}}$.

Tabla 10. Escala Pentaria Base en la Evaluación en la CVC en la EEec

\begin{tabular}{|c|l|}
\hline Alfa-Corte & \multicolumn{1}{|c|}{ Reglas Lexicográficas } \\
\hline 0,00 & Falso \\
\hline 0,25 & Más falso que verdadero \\
\hline 0,50 & Ni verdadero ni falso \\
\hline 0,75 & Más verdadero que falso \\
\hline 1 & Verdadero \\
\hline
\end{tabular}

Fuente: Adaptada de [23].

TABla 11. InFormación Base para La Evaluación TÉCNICA-FinanCIERA

\begin{tabular}{|c|c|}
\hline AÑO & FCTNA para la UCEM con soporte en NBT \\
\hline 0 & $\$(138,697.140)$ \\
\hline 1 & $(\$ 2,106.680, \$ 2,179.470, \$ 2,201.270)$ \\
\hline 2 & $(\$ 26,003.130, \$ 26,901.640, \$ 27,170.660)$ \\
\hline 3 & $(\$ 75,651.530, \$ 78,265.600, \$ 79,048.260)$ \\
\hline 4 & $(\$ 80,041.990, \$ 82,807.770, \$ 83,635.850)$ \\
\hline 5 & $(\$ 188,644.910, \$ 195,163.370, \$ 197,115.000)$ \\
\hline
\end{tabular}

Fuente: Autor.
La tabla 12, recoge los intervalos de confianza en los que se soporta la valoración del VPN $\mathrm{NIA}_{\mathrm{AI}}$, los cuales, resultan de aplicar la expresión (23) a la información suministrada en la tabla 11. Además, la valoración del $\mathrm{VPN}_{\mathrm{AIA}}$ se presenta en la tabla 13, en la cual, se empleó a (24) combinada con la información que se recogen en las tablas 10 y 12 .

El FCTN $\mathrm{FIA}_{\mathrm{AI}}$ de la estrategia de integración del tipo ganar-ganar soportada en el mecanismo de CVC, que lleva por nombre $\mathrm{UCE}_{\mathrm{M}}$ presentado en la tabla 11, fue diseñado mediante el empleo de (21) a (22).

Tabla 13. Niveles de Presunción de los NbT Para el VPNAia

\begin{tabular}{|c|c|c|}
\hline ALFA-CORTE & VPNALI & VPNALS \\
\hline 0,00 & $\$ 21,809.310$ & $\$ 29,016.000$ \\
\hline 0,25 & $\$ 23,195.850$ & $\$ 28,600.870$ \\
\hline 0,50 & $\$ 24,582.390$ & $\$ 28,185.730$ \\
\hline 0,75 & $\$ 25,968.930$ & $\$ 27,770.600$ \\
\hline 1,00 & $\$ 27,355.470$ & $\$ 27,355.470$ \\
\hline & Fuente: Autor. \\
\hline
\end{tabular}

En la tabla 13, se observa que la estrategia de integración del tipo ganar-ganar diseñada desde la óptica del AGV, con soporte en la $\mathrm{UCE}_{\mathrm{M}}$ en Condiciones de Incertidumbre y Ambigüedad, CVC para los agentes que hacen parte de la $\mathrm{EE}_{\mathrm{EC}}$ bajo las condiciones diseñadas mediante SCAMPER con paradigma holónico, evaluación técnico-financiera basada en el siguiente NBT:

\section{VPNA [\$ 21.809.310; \$27.355.470; \$ 29.016.000]}

Donde el máximo nivel de presunción de CVC para la EE es de $\$ 27.355 .470$, en el peor de los casos la EE CVC por tan sólo \$21.809.310, y en el más optimista CVC por \$29.016.000. Más aun, en la tabla 14 se presenta las $\mathrm{TVR}_{\mathrm{A}}$ que ratifican la CVC para la EEEC, en cuestión.

Tabla 12. Intervalos de los FCtn con Alfa-Cortes

\begin{tabular}{|c|c|}
\hline FCTNA & INTERVALO de CONFIANZA \\
\hline 1 & {$[\$ 2,106.680+\$ 72.790 a, \$ 2,201.270-\$ 21.800 a]$} \\
\hline 2 & {$[\$ 26,003.130+\$ 898.510 a, \$ 27,170.660-\$ 269.020 a]$} \\
\hline 3 & {$[\$ 75,651.530+\$ 2,614.070 a, \$ 79,048.260-\$ 782.660 a]$} \\
\hline 4 & {$[\$ 80,041.990+\$ 2,765.780 a, \$ 83,635.850-\$ 828.080 a]$} \\
\hline 5 & {$[\$ 188,644.910+\$ 6,518.460 a, \$ 197,115.000-\$ 1,951.630 a]$} \\
\hline
\end{tabular}

Fuente: Autor. 
Tabla 14. Niveles de Presunción de los NBT para el TVRaia

\begin{tabular}{|c|c|c|}
\hline ALFA-CORTE & $\begin{array}{c}\text { TVRALI } \\
\text { (E.A.) }\end{array}$ & $\begin{array}{c}\text { TVRALS } \\
\text { (E.A.) }\end{array}$ \\
\hline 0,00 & $26,85 \%$ & $27,97 \%$ \\
\hline 0,25 & $27,07 \%$ & $27,91 \%$ \\
\hline 0,50 & $27,29 \%$ & $27,84 \%$ \\
\hline 0,75 & $27,50 \%$ & $27,78 \%$ \\
\hline 1,00 & $27,72 \%$ & $27,72 \%$ \\
\hline
\end{tabular}

Así mismo, el NBT que se cita a continuación:

TVRA [26.85\% E.A; $27.72 \%$ E.A; $27.97 \%$ E.A]

Ratifica, otra vez, más la CVC, para quienes conforman la $\mathrm{EE}_{\mathrm{EC}}$ vía el diseño de la estrategia del tipo ganar-ganar con soporte en el Método SCAMPER con paradigma holónico; donde el máximo nivel de presunción de CVC para la EE es del 27.72\% E.A, en el peor de los casos la EE CVC por tan sólo el $26.85 \%$ E.A, y en el más optimista CVC por el $27.97 \%$ E.A.

\section{Conclusiones}

El presente estudio, concuerda con los postulados de [1] y [19] en lo referente, al cómo, se debe: i) Abordar una problemática en logística urbana. Sugiere [1] el Triángulo de las Tres T como metodología de trabajo, a saber: Teoría-Tecnología-Técnica; y ii) Solucionar una problemática en logística urbana. Se ha combinado, por parte de [19] dos de las cuatro herramientas de gestión, que para tal fin, usa el profesional de la ingeniería industrial, la Investigación de Operaciones y la Ingenieria Económica. Más aun, esta investigación no sólo aborda y soluciona de igual manera su caso de aplicación sino también lo robustece, al concebir inversiones en valor de vida, por un lado, y al vincular las otras dos herramientas de gestión, tales como: La Gestión Tecnológica y la Optimización Estadística, por el otro.

Por otra parte, esta investigación comparte el mismo punto de vista asumido por [3], con respecto al diseño operacional de organizaciones sostenibles bajo el paradigma holónico, que posibilita la concepción de la $\mathrm{EE}_{\mathrm{EC}}$ de tipo $\mathrm{DUM}_{\mathrm{HAS}}$ con ajuste por variedad en el entorno, tanto en tiempo como en espacio. Es decir, la $\mathrm{EE}_{\mathrm{EC}}$ diseñada bajo los principios del paradigma holónico, les permite a los holones, por un lado, la automejora, y por el otro lado, la autoorganizan, a la vez, que garantiza independencia económica, autonomía administrativa, y respecto por su idiosincrasia e cultural organizacional [30], al permitir el diseño de estrategias de integración del tipo ganar-ganar, basadas en procesos tanto colaborativos como cooperativos [3].
Con respecto al diseño de la EEEC en función del AGV en ambientes urbanos, esta investigación no sólo comparte con [1] la directriz Localización-AsignaciónTransporte-Inventarios, sino que además, le adiciona el concepto de Integración tanto vertical como horizontal, dándole al enfoque de diseño, un perfil estratégico de configuración de Redes de Suministro Urbano con enfoque Sostenible ( $\mathrm{RdS}_{\mathrm{US}}$ ), por medio del cual, se demuestra que el CIF requerido por el acuerdo contractual y vinculante entre los agentes que se coalicionan CVC durante su vigencia.

En términos generales, el diseño de una RdSUS debe estar concebida sobre la base de los elementos que pueden ser: i) Sustituidos; ii) Combinados; iii) Adaptados; iv) Modificados; v) Permutados; vi) Eliminados; y vii) Reordenados; Con el propósito de mejorar una medida de desempeño de la EE, hacer más sencillo la configuración de la EE o perfeccionar otros beneficios para la EE, y en lo posible, valorado en términos de CVC en condiciones de incertidumbre e ambigüedad.

A partir de los hallazgos del presente estudio, cabe resaltar la pertinencia de abordar a futuro entre otros el siguiente interrogante: ¿Cómo mejora el diseño de la estrategia de administración del inventario conjunto en estado de incertidumbre y ambigüedad, con respecto al agente generador de viajes en la empresa extendida urbana de economía compartida, al incorporar a la MIEL $_{\mathrm{CVC}}$ un diseño factorial con soporte en una función de deseabilidad? así como, los aspectos relacionados con su verificación en un modelo de regresión no lineal múltiple; con la finalidad de Viabilizar, Elegibilizar y Priorizar los factores que influyen en forma significativa en la CVC [30]-[31].

También es de interés del autor configurar la UCE en condiciones de volatilidad, incertidumbre, complejidad y ambigüedad, en una Red de Suministros Urbana Holónica de Economía Compartida con respecto al AGV, basada en un Analisis de Eficiencia Técnico-Económica y Financiera con orientación a la CVC en forma Sostenible, en donde, la definición de las características, que soporta la estrategia de negocio del tipo ganar-ganar, se haga vía contraste en una función de perdida sobre un abanico de estrategias experimentales [30]-[31].

\section{FinANCIAMIENTO}

Artículo de investigación científica derivado del proyecto de investigación "Metodología para la Coordinación de Inventarios en una Red de Suministros Urbana”, comprometido en el Marco del Periodo Sabático con Resolución 4812 de Diciembre 05 de 2017 otorgado por la Rectoría de la Universidad Militar Nueva Granada. Fecha de inicio: Enero 20 de 2018, Fecha de finalización: Enero 19 de 2019. 


\section{Agradecimientos}

A mi director de tesis doctoral, el profesor doctor Wilson Adarme Jaimes por su gran ayuda y colaboración en cada momento de consulta y soporte, durante la construcción de este producto de investigación.

\section{REFERENCIAS}

[1] W. Adarme-Jaimes, "Notas de clase de la asignatura: Logística Aplicada a Redes de Distribución y Transporte", Maestría en Ingeniería, Dept. Ing. de SI., Ing., Universidad Nacional de Colombia, Bogotá D.C, 2018.

[2] M. D. Arango-Serna, J. W. Branch-Bedoya and J. E. Londoño-Salazar, "Enterprise architecture as tool for managing operational complexity in organizations", Dyna, vol. 81, no.185, pp. 219-226, May. 2014. http:// dx.doi.org/10.15446/dyna.v81n185.41928

[3] M. J. Ávila-Gutiérrez, F. Aguayo-González, M. MarcosBárcena, J. Lama-Ruiz and M. Peralta-Álvarez, "Reference holonic architecture for sustainable manufacturing enterprises distributed", Dyna, vol. 84, no. 200, pp. 160-168, Jan. 2017. http://dx.doi.org/10.15446/dyna. v84n200.53095

[4] A. Bernardello, M. Bianco, M. Casparri, J. García y S. Olivera, "Modelos dinámicos discretos", en Matemáticas para Economistas con Excel y Matlab. Buenos Aires: Omicron System, 2004, pp. 328-329.

[5] J. Browne and J. Zhang, "Extended and virtual enterprises - similarities and differences", International Journal of Agile Management Systems, vol. 1, no. 1, pp.30-36, Apr. 1999. https://doi.org/10.1108/14654659910266691

[6] J. J. Buckley, "The fuzzy mathematics of finance", Fuzzy sets and systems, vol. 21, no. 3, pp. 257-273, Mar. 1987. https://doi.org/10.1016/0165-0114(87)90128-X

[7] V. B. Campos, y I. C. Melo, Análisis de la demanda de transporte de carga en área urbana bajo el punto de vista de la producción y de la atracción de viajes, Zaragoza, España: VI-CIT, 2004.

[8] S. Chopra and P. Meind, Supply chain management: strategy, planning and operation. Evanston-Illinois, USA: Pearson Education, 2016.

[9] T. G. Crainic, City Logistics. Montreal, Canadá: CIRRELT, 2008.

[10] L. Cuatrecasas, Organización de la producción y dirección de operaciones: Sistemas actuales de gestión eficiente y competitiva. Madrid, España: Ediciones Díaz de Santos, 2011.

[11] Conpes 3819. Política nacional para consolidar el sistema de ciudades en Colombia. Consejo Nacional de Política Económica y Social.Departamento Nacional de Planeación. 21-10-2014.

[12] B. L. Gil, y M. A. Vieira, "Análise de geração de viagens de carga em área urbana", XXIX-CNPTA, Congresso de Pesquisa e Ensino em Transportes da anpet, Ouro Preto, 2015, p. 10.

[13] B. Fleischmann, "The impact of the number of parallel warehouses on total inventory". OR Spectrum, vol. 38, no. 4, pp. 899-920, Apr. 2016. https://doi.org/10.1007/ s00291-016-0442-2

[14] M. G. Fiestras-Janeiro, I. García-Jurado, A. Meca, and M. A. Mosquera, "Cost allocation in inventory transportation systems" Top. [Online], vol. 20, no. 2, pp. 397410, Jul. 2012. https://doi.org/10.1007/s11750-011-02077

[15] M. G. Fiestras-Janeiro, I. García-Jurado, A. Meca, and M. A. Mosquera, "Centralized inventory in a farming community". Journal of Business Economics, vol. 84, no. 7, pp. 983-997, Oct. 2014. https://doi.org/10.1007/ s11573-014-0710-z
[16] J. P. García, C. Carboneras, J. Albarracín, y J. García, Gestión de stocks de demanda independiente, Valencia, España: Editorial Universitat Politècnica de ValènciaUPV, 2004, pp. 74-75.

[17] D. B. Gillies, "Some theorems on n-person games", Dissertation Ph.D, Dept. OR and FE, Eng., Princeton Univ., Princeton, N.J, USA, 1953.

[18] R. P. Gillies, The Cooperative Game Theory of Networks and Hierarchies. Belfast, UK: Springer Science \& Business Media, 2010.

[19] J. Gonzalez-Feliu, F. Semet and J. Routhier, Sustainable Urban Logistics: Concepts, Methods and Information Systems. Heidelberg, Germany: Springer, 2014.

[20] M. Gutiérrez y V. Cantillo, "Una propuesta para la modelación de transporte de carga interregional basado en el análisis de productos", en IX-SITT Simposio de Ingeniería de Tránsito y Transporte. Villa de Leyva, Nov. 19-21, 2009, p. 10

[21] J. Holguín-Veras, C. Wang, M. Browne, S. Darville and J. Wojtowicz, "The New York City off-hour delivery project: Lessons for city logistics", Procedia-Social and Behavioral Sciences, vol. 125, pp. 36-48, Mar. 2014. https://doi.org/10.1016/j.sbspro.2014.01.1454

[22] R. Jordán, L. Riffo y A. Prado, "La producción socioespacial de las ciudades latinoamericanas y la sostenibilidad urbana”, en Desarrollo sostenible, urbanización y desigualdad en América Latina y el Caribe: Dinámicas y desafíos para el cambio estructural, Santiago de Chile, Chile: Naciones Unidas, 2017, p. 215.

[23] A. Kaufmann y J. Gil-Aluja, Introducción de la teoría de los subconjuntos borrosos a la gestión de las empresas. Vigo, España: Editorial Milladoiro S.I, 1986.

[24] K. C. Tan, "An Innovation in Last-Mile Delivery", in: TA-Q-BIN: Service Excellence and Innovation in Urban Logistics. Singapore: Springer, 2015. https://doi. org/10.1007/978-981-287-673-7_4

[25] D. H. Maister, "Centralization of Inventories and the Square Root Law", International Journal of Physical Distribution, vol. 6, no. 3, pp. 124-134, Dec. 1976. https://doi.org/10.1108/eb014366

[26] M. Mauleón, "Embalaje y transporte", en Transporte, Operadores, Redes. Madrid, España: Ediciones Díaz de Santos, 2013, p. 155 .

[27] L. A. Metzler, "The nature and stability of inventory cycles", The Review of Economics and Statistics, vol. 23, no. 3, pp. 113-129, Aug. 1941. http://www.jstor.org/stable/1927555

[28] M. Navarrete-Peñuela, "Desarrollo urbano sustentable: el gran desafío para américa latina y los preparativos para Hábitat III". Revista Luna Azul, vol. 45, no. 1, pp. 123-149, Jul. 2017. https://doi.org/10.17151/ luaz.2017.45.8

[29] L. K. De Oliveira, R. Magalhães, C. De Fátima y D. Gonçalves, "Modelo de geração de viagens de carga em áreas urbanas: um estudo para bares, restaurantes e supermercados", Revista Transportes, vol. 24, no. 2, pp. 53-67, Mar. 2016. https://doi.org/10.14295/transportes. v24i2.1058

[30] O. Palacio-León, "Metodología para la Coordinación de Inventarios en una Red de Suministros Urbana”, [TD en Ejecución], Dpto. Ing. Industrial, Ing., UC, Valencia, Venezuela, 2019

[31] O. Palacio-León, "Propuesta metodológica para la evaluación financiera de proyectos inmobiliarios de tipo industrial", [TD en Ejecución], Dpto. Proyectos, UNINIMéxico, Campeche, México, 2019.

[32] A-W. Scheer, Architektur integrierter Informationssysteme: Grundlagen der Unternehmensmodellierung, Heidelberg, Germany: Springer, 1992

[33] O. Serrat, "The SCAMPER Technique", In: Knowledge Solutions: Tools, Methods, and Approaches to Drive Organizational Performance, Singapore: Springer, 2017, pp. 311-314. 
[34] E. Taniguchi, T. Fwa and R. Thompson, Urban Transportation and Logistics: Health, Safety, and Security Concerns, Boca Raton, USA: CRC Press, 2014.

[35] R. J. Tersine, "Independent demand systems: Deterministic models", In: Principles of inventory and materials management. New York, USA: Prentice-Hall, 1994, pp. 137-139.

[36] M. S. Viana, y J. P. Delgado, "Análise espacial da demanda de transporte urbano de mercadorías na área central da cidade de salvador visando a. aplicabilidade de estratégias de logística urbana”, XXIX-CNPTA Congresso Nacional de Pesquisa em Transporte da Anpet. Ouro Preto, 2015, p. 12.

[37] W. K. Viscusi, "The value of life: estimates with risks by occupation and industry", Economic Inquiry, vol. 42, no. 1, pp. 29-48, Mar. 2004. https://doi.org/10.1093/ei/ cbh042

[38] K. Yang, L. Yang and Z. Gao, "Hub-and-spoke network design problem under uncertainty considering financial and service issues: A two-phase approach", Information Sciences, vol. 402, pp. 15-34, Sep. 2014. https://doi. org/10.1016/j.ins.2017.03.022

[39] L. A. Zadeh, "Fuzzy sets", Information and Control, vol. 8, no. 3, pp. 338-353, Jun. 1965. https://doi.org/10.1016/ S0019-9958(65) $90241-\mathrm{X}$
Oscar Palacio-León es Ing. Industrial, Esp. en Evaluación y Desarrollo de Proyectos, MSc. en Investigación Operativa y Estadística, MSc. en Ingeniería con mención en Ingeniería Industrial, Doctorando en Ingeniería en la UC (Venezuela) y Doctorando en Proyectos en la UNINI (México). En la actualidad se desempeña como profesor asociado en el Programa de Ingeniería Industrial de la Universidad Militar Nueva Granada. Sus principales áreas de investigación son: Diseño de Redes Urbanas de Suministro y Evaluación de Proyectos Inmobiliarios de Tipo Industrial, con publicación de resultados en revistas indexadas. Consultor en planeación estratégica de centros de distribución urbanos de mercancías para Mipymes y Multinacionales. https://orcid.org/00000003-2417-311X 